\title{
Fracture repair requires TrkA signaling by skeletal sensory nerves
}

\author{
Zhu Li, ${ }^{1,2}$ Carolyn A. Meyers, ${ }^{3}$ Leslie Chang, ${ }^{3}$ Seungyong Lee, ${ }^{3}$ Zhi Li, ${ }^{1}$ Ryan Tomlinson, ${ }^{4}$ Ahmet Hoke, ${ }^{5}$ \\ Thomas L. Clemens, ${ }^{1,2}$ and Aaron W. James ${ }^{3}$ \\ 'Department of Orthopaedics, Johns Hopkins University, Baltimore, Maryland, USA. ${ }^{2}$ Baltimore Veterans Administration Medical Center, Baltimore, Maryland, USA. ${ }^{3}$ Department of Pathology, Johns Hopkins \\ University, Baltimore, Maryland, USA. ${ }^{4}$ Thomas Jefferson University, Philadelphia, Pennsylvania, USA. ${ }^{5}$ Department of Neuroscience, Johns Hopkins University, Baltimore, Maryland, USA.
}

Bone is richly innervated by nerve growth factor-responsive (NGF-responsive) tropomyosin receptor kinase A-expressing (TrKa-expressing) sensory nerve fibers, which are required for osteochondral progenitor expansion during mammalian skeletal development. Aside from pain sensation, little is known regarding the role of sensory innervation in bone repair. Here, we characterized the reinnervation of tissue following experimental ulnar stress fracture and assessed the impact of loss of TrkA signaling in this process. Sequential histological data obtained in reporter mice subjected to fracture demonstrated a marked upregulation of NGF expression in periosteal stromal progenitors and fracture-associated macrophages. Sprouting and arborization of CGRP+TrkA+ sensory nerve fibers within the reactive periosteum in NGF-enriched cellular domains were evident at time points preceding periosteal vascularization, ossification, and mineralization. Temporal inhibition of TrkA catalytic activity by administration of 1 NMPP1 to TrkA ${ }^{\mathrm{F} 92 \mathrm{~A}}$ mice significantly reduced the numbers of sensory fibers, blunted revascularization, and delayed ossification of the fracture callus. We observed similar deficiencies in nerve regrowth and fracture healing in a mouse model of peripheral neuropathy induced by paclitaxel treatment. Together, our studies demonstrate an essential role of TrkA signaling for stress fracture repair and implicate skeletal sensory nerves as an important upstream mediator of this repair process.

\section{Introduction}

Mature bone is richly innervated by sensory nerves that infiltrate the developing axial and appendicular skeleton from the dorsal root $(1,2)$. The most familiar role of sensory nerves is to transmit nociceptive signals, which become profoundly apparent following bone fracture. The central mediator of bone pain is nerve growth factor (NGF), which transmits nociceptive signals either by direct activation of tropomyosin receptor kinase A-expressing (TrkAexpressing) sensory neurons or through indirect mechanisms, which enhance the response of other nociceptive pathways $(3,4)$.

In addition to pain sensation, a large body of literature supports an evolutionarily conserved role of TrkA sensory nerves in organogenesis and tissue regeneration (5). During the development of the peripheral nervous system, tissues dictate the amount and type of innervation they require by secreting neurotrophic factors, which promote neuronal survival (6). The prototypic target cell-derived neurotrophic factor NGF supports survival of developing neurons by activating one of the Trk family of receptors (7). Following activation, phosphorylation of TrkA (p-TrkA) at the axon tip or cell body can activate SHC, PI-3 kinase, and PLC- $\gamma 1$ to initiate the Ras

Authorship note: Zhu Li, CAM, and LC share first authorship.

Conflict of interest: The James laboratory receives research support from Musculoskeletal Transplant Foundation (MTF) Biologics.

Copyright: (c) 2019, American Society for Clinical Investigation.

Submitted: February 27, 2019; Accepted: August 20, 2019; Published: October 22, 2019

Reference information: / Clin Invest. 2019;129(12):5137-5150.

https://doi.org/10.1172/JCl128428. signaling pathway (8). Because NGF is received by sensory nerve axons distant to their cell body, a unique mechanism has evolved to shuttle signals to the neuronal cell body. Following activation, neurotrophin-receptor complexes are internalized by endocytosis and form specialized signaling vesicles that undergo longdistance, retrograde transport from the distal axon to the cell body via a microtubule-based transport mechanism (9). In the developing mouse skeleton, TrkA sensory neurons innervate the perichondrium of the developing long bones at sites of NGF production where they are required for subsequent bone progenitor cell differentiation and mineralization (10). In lower vertebrates, an analogous paradigm for sensory nerve innervation appears to operate during tissue regeneration. For example, the ability of starfish and certain amphibians to regenerate entire appendages depends on sensory or motor neurons that are believed to drive subsequent assembly of vascular and tissue components (5). The few studies conducted to date in rodents show that NGF is markedly upregulated acutely following bone injury (11-13) and that ablation of nerves by nonspecific surgical means impairs fracture healing $(14,15)$. However, whether TrkA signaling by sensory nerves is required for mammalian fracture repair and the cellular processes affected by these signals in this setting are unclear.

In this study, we conducted a detailed assessment of the cellular events accompanying reinnervation of bone following experimental stress fracture in mice. Using compound mice carrying mutant TrkA alleles and sensitive fluorescent sensory nerve reporters, we demonstrate that TrkA signaling is required for sensory nerve regeneration and for normal fracture repair. Similar deficiencies 
in nerve regrowth and fracture healing were observed in a mouse model of peripheral neuropathy induced by paclitaxel (PTX). Our results document in detail the sequence of cellular events during reinnervation of a fracture callus and demonstrate the requirement for TrkA-expressing sensory nerves in bone healing.

\section{Results}

NGF expression is acutely upregulated following fracture. We performed cyclic fatigue end-loading to achieve a nondisplaced stress fracture of the ulnar mid-diaphysis (Figure 1A). 3D micro-CT $(\mu \mathrm{CT})$ reconstructions (Figure $1 \mathrm{~B})$, coronal cross-sectional images (Figure 1C), and axial cross sections (Figure 1D) illustrated the normal timeline of stress fracture healing over a 56-day period. Woven bone formation was first observed by $\mu \mathrm{CT}$ on day 7 after fracture, the hard callus size was largest on day 14 after fracture, and remodeling and corticalization of the callus was most apparent at the study endpoint of day 56 after fracture. $\mu \mathrm{CT}$ based quantification of the fracture callus demonstrated an increase in bone volume (BV) by day 7 and was highest on the day-14 time point (Figure 1E). Fractional BV to tissue volume (BV/TV) rose over time, starting on day 7 after injury, and approached $100 \%$ by day 28 after fracture (Figure 1F). Bone mineral density (BMD) likewise increased over time and approached the density of uninjured ulnar cortex by day 56 after injury (Figure $1 G$ ).

The temporo-spatial domains of NGF expression were next characterized over the same 56-day period, using a previously validated NGF-eGFP reporter animal (Figures 2 and 3 and ref. 16). Fluorescent microscopic images are presented as tile scans to encompass a longitudinal cross section of the fracture callus with underlying cortex, as well as corresponding high-magnification images of reporter activity and H\&E staining to provide cellular detail (Figure 2, A-U). A linear, weak NGF reporter was present within the uninjured periosteum, but not the underlying cortical osteocytes (Figure 2, A-C). At time points preceding callus ossification, the majority of cells constituting the periosteal callus were NGF reporter positive (Figure 2, D-I; days 1-3). At time points of most robust bone matrix deposition, the majority of reporter-positive cells were bone-lining cells and bone-entombed cells within the hard callus (Figure 2, J-O; days 7-14). At later times corresponding to corticalization of the hard callus, NGF reporter activity waned, and cells within bone matrix were largely reporter negative (Figure 2, P-U; days 28-56). At all time points, native cortical osteocytes remained NGF reporter negative. Quantitative analysis revealed an increase in relative NGF-eGFP reporter activity within the periosteal callus, which was highest on day 3 and returned to baseline over the study period (Figure 2V).

Next, we performed immunohistochemical staining of NGFeGFP reporter sections with cell type-specific antibodies. Sections were first interrogated 3 days after injury - a time point before ossification and at the peak of reporter activity levels (Figure 3, A-H). Results revealed dual sources of NGF within a nascent callus, including periosteal mesenchymal cells, which showed strong expression of vimentin and PDGFR $\alpha$ (Figure 3, A and B), as well as patchy expression of PDGFR $\beta$ (Figure $3 \mathrm{C}$ ). CD $45^{+}$inflammatory cells were noted as a second source of reporter activity (Figure 3D). The primary inflammatory cell type with reporter activity was $\mathrm{F} 4 / 80^{+}$macrophages (Figure $3 \mathrm{E}$ ), whereas other inflamma- tory cell types, including $\mathrm{Ly}-6 \mathrm{G}^{+}$polymorphonuclear leukocytes and $\mathrm{CD} 117^{+}$mast cells, did not show significant overlap with NGFeGFP reporter activity (Figure 3, F and G). Quantitative analysis of serial sections among e $\mathrm{GFP}^{+}$cells types within an early fracture callus revealed that the majority of reporter positive cells were periosteal/stromal cells, followed by macrophages (Figure $3 \mathrm{H}$ ). We next analyzed reporter sections on day 14 after injury - a time point at the peak of bone volume within the healing tissue (Figure $3, \mathrm{I}-\mathrm{O})$. Here, osteocalcin $\left(\mathrm{OCN}^{+}\right)$bone-lining osteoblasts appeared to be the primary source of reporter activity (Figure 3I), whereas occasional tartrate-resistant acid phosphatase-positive (TRAP ${ }^{+}$) osteoclasts were also reporter positive (Figure 3J). Neither CD45 inflammatory cells (Figure $3 \mathrm{~K}$ ), CD31 ${ }^{+}$endothelial cells (Figure $3 \mathrm{~L}$ ), or PDGFR $\beta^{+}$pericytes (Figure $3 \mathrm{M}$ ) were a significant cell type with NGF-eGFP reporter activity. Quantitative analysis among $\mathrm{eGFP}^{+}$cells types within a hard callus confirmed that the majority of reporter-positive cells were osteoblastic (Figure 30).

Reinnervation of the fracture callus by sensory nerves precedes angiogenesis at sites of new bone formation. First, we examined the extent to which periosteal nerve fibers were disrupted after stress fracture. For this purpose, we performed $\beta$ III tubulin (TUBB3) whole-mount IHC on the ulna diaphysis before and after fracture (Figure 4, A and B). As expected of a nondisplaced stress fracture, the results showed no clear disruption of longitudinal $\mathrm{TUBB}^{+}$nerve fibers as they crossed the fracture site. Next, we visualized and quantified the reinnervation of the fracture callus in mice carrying the pan-neuronal transgene Thy1-YFP over an 8-week healing period (17). In images of longitudinal cross sections, we observed delicate single nerve fibers coursing longitudinally within the uninjured periosteum (Figure 4C). At early time points preceding ossification, we observed prominent dendrite sprouting and duplication coursing longitudinally over the reactive periosteum (Figure 4, D and E). At later time points during and after bone matrix deposition, yellow fluorescent proteinpositive $\left(\mathrm{YFP}^{+}\right)$nerve fibers were more prominent than in uninjured bone but were reduced in frequency and often confined to the outer fibrous capsule of the hard callus, with infrequent single nerve fibers found coursing between the interstices of bone trabeculae (Figure 4, F-I). Quantification of Thy1-YFP reporter activity within the fracture callus was done over a 56-day period after injury (Figure 4J). The results showed that callus innervation was higher than in uninjured periosteum at all time points, with the highest nerve fiber frequency seen on days 1 and 3 after injury time points preceding bone matrix deposition (Figure 4J).

Next, we used TrkA-LacZ reporter animals (18) to assess whether fracture-associated neuronal sproutings were also TrkAexpressing fibers (Supplemental Figure 1; supplemental material available online with this article; https://doi.org/10.1172/ JCI128428DS1). As with Thy1-YFP reporter activity, we found that uninjured periosteum housed inconspicuous $\mathrm{LacZ}^{+}$nerve fibers (Supplemental Figure 1A). On day 14, the periphery of the hard callus showed a substantial increase in $\mathrm{LacZ}^{+}$nerve fiber numbers (Supplemental Figure 1B). We verified the specificity of X-gal staining using analogous sections from $\mathrm{LacZ}^{-}$fracture sites (Supplemental Figure 1C). We quantified the increased frequency of TrkA-expressing nerve fibers compared with the uninjured ulnar periosteum (Supplemental Figure 1D). 


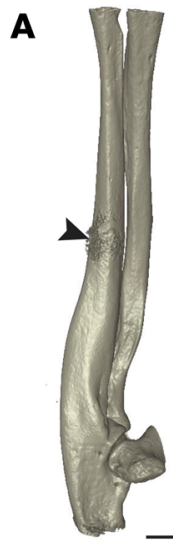

B Uninjured
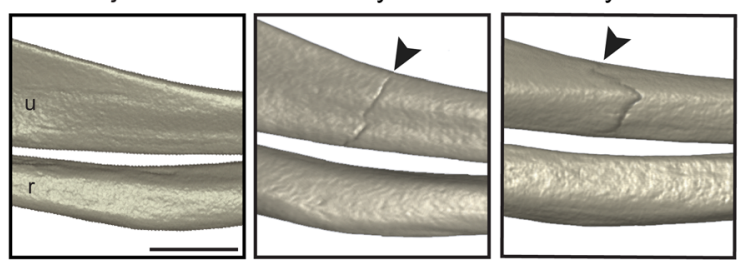

E

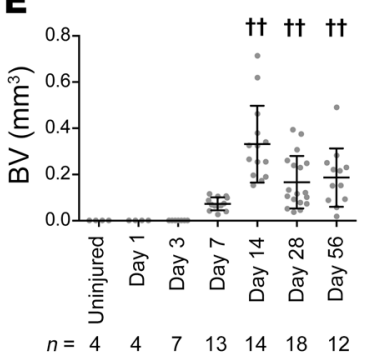

F

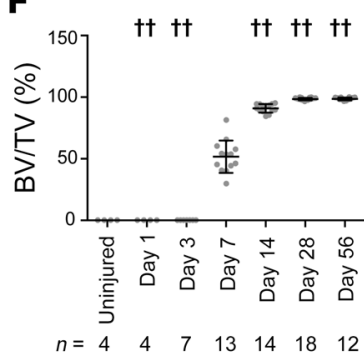

G

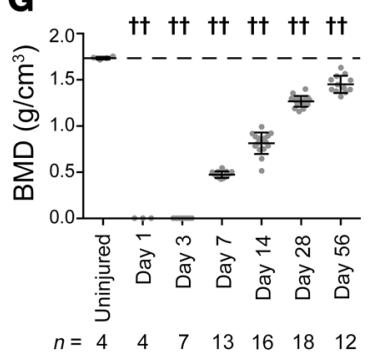

C
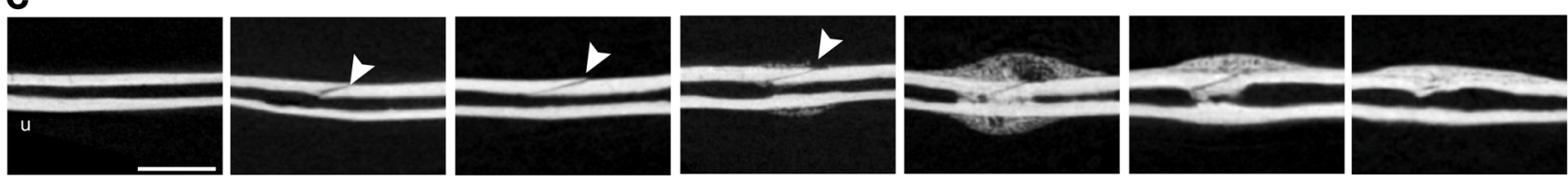

D
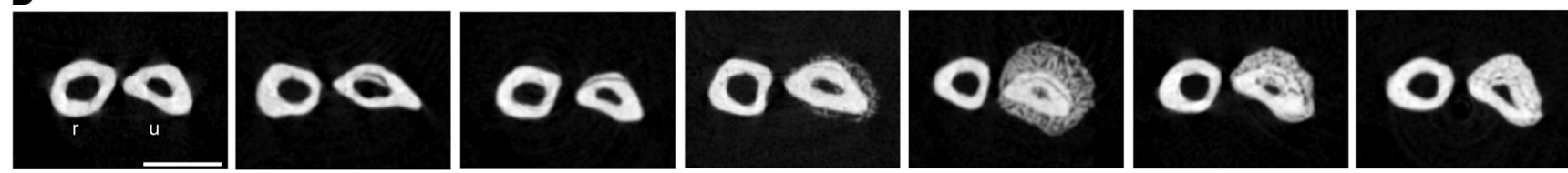

Figure 1. Fatigue end-loading induces ulnar stress fracture. (A) $\mu \mathrm{CT} 3 \mathrm{D}$ reconstruction of a mouse forelimb showing a mid-diaphyseal healing fracture of the ulna (a representative image from day 7 is shown). Scale bar: $500 \mu \mathrm{m}$. (B) $\mu \mathrm{CT}$ 3D reconstructions, (C) coronal cross-sectional images, and (D) axial cross-sectional images at serial time points after stress fracture from days 1-56. Arrowheads indicate fracture sites. The uninjured forelimb is shown for comparison. Black scale bar: $500 \mu \mathrm{m}$; white scale bars: $1 \mathrm{~mm}$. (E-G) Quantitative analysis of $\mu \mathrm{CT}$ imaging on days 1-56 after stress fracture. (E) BV of callus; (F) BV/TV of callus; and (C) BMD of callus. Dashed line indicates the mean BMD of uninjured ulnar cortical bone at the mid-diaphysis. For all graphs, each dot represents a single sample, with each sample number indicated below. Data are expressed as the mean $\pm \mathrm{SD}$. ${ }^{+t} P<0.01$ versus the day-7 time point $(\mathbf{E}$ and $\mathbf{F}$ ) or versus the uninjured control (G), by 1-way ANOVA with post hoc Newman-Keuls test. $u$, ulna; r, radius.

Both sensory and peptidergic fibers and sympathetic fibers found within the periosteum express TrkA (2). To define the predominant nerve type affected after fracture, we next assessed the immunohistochemical detection of CGRP ${ }^{+}$peptidergic fibers and $\mathrm{TH}^{+}$sympathetic fibers on sections from Thy1-YFP reporter mice (Figure 4, K-R). In representative images, Thy1 reporter activity is shown in green, whereas the overlap between immunohistochemical stains and Thy1 reporter activity is shown in yellow. Both CGRP ${ }^{+}$peptidergic fibers and $\mathrm{TH}^{+}$sympathetic fibers were found within uninjured periosteum and partially overlapped with Thy1 reporter activity (Figure 4, $\mathrm{K}$ and L). We performed similar experiments on Thy1 reporter sections of ulnar fractures on days 3 and 14 after injury and assessed quantitative changes in fiber frequency (Figure 4, M-R). We observed a conspicuous increase in $\mathrm{CGRP}^{+}$fibers on post-fracture day 3, which was reduced but remained elevated over baseline levels on post-injury day 14 (Fig- ure $4, \mathrm{M}-\mathrm{O}$; $\mathrm{CGRP}^{+}$nerve fibers constituted $83.0 \%$ and $87.5 \%$ of total nerve fibers within the callus on days 3 and 14, respectively). We observed a less prominent increase in $\mathrm{TH}^{+}$fiber frequency on day 3 after injury that returned to levels comparable to those in uninjured controls on day 14 after injury (Figure 4, $\mathrm{P}-\mathrm{R} ; \mathrm{TH}^{+}$nerve fibers constituted $17.0 \%$ and $12.5 \%$ of total nerve fibers within the callus on days 3 and 14, respectively). Thus, and in line with histologic observations, the change in $\mathrm{CGRP}^{+}$fiber frequency after injury more closely mirrored that of $\mathrm{YFP}^{+}$total nerve fibers, whereas $\mathrm{TH}^{+}$fibers were less prominently altered after stress fracture.

Skeleton-associated nerves and blood vessels often follow similar paths within bone tissue $(1,19)$ and are thought to influence the trajectory of one another as they grow toward target tissues $(20,21)$. We assayed blood vessel distribution and frequency with CD31 staining at serial time points after fracture, again using tissue sections from Thy1-YFP reporter animals (Figure 

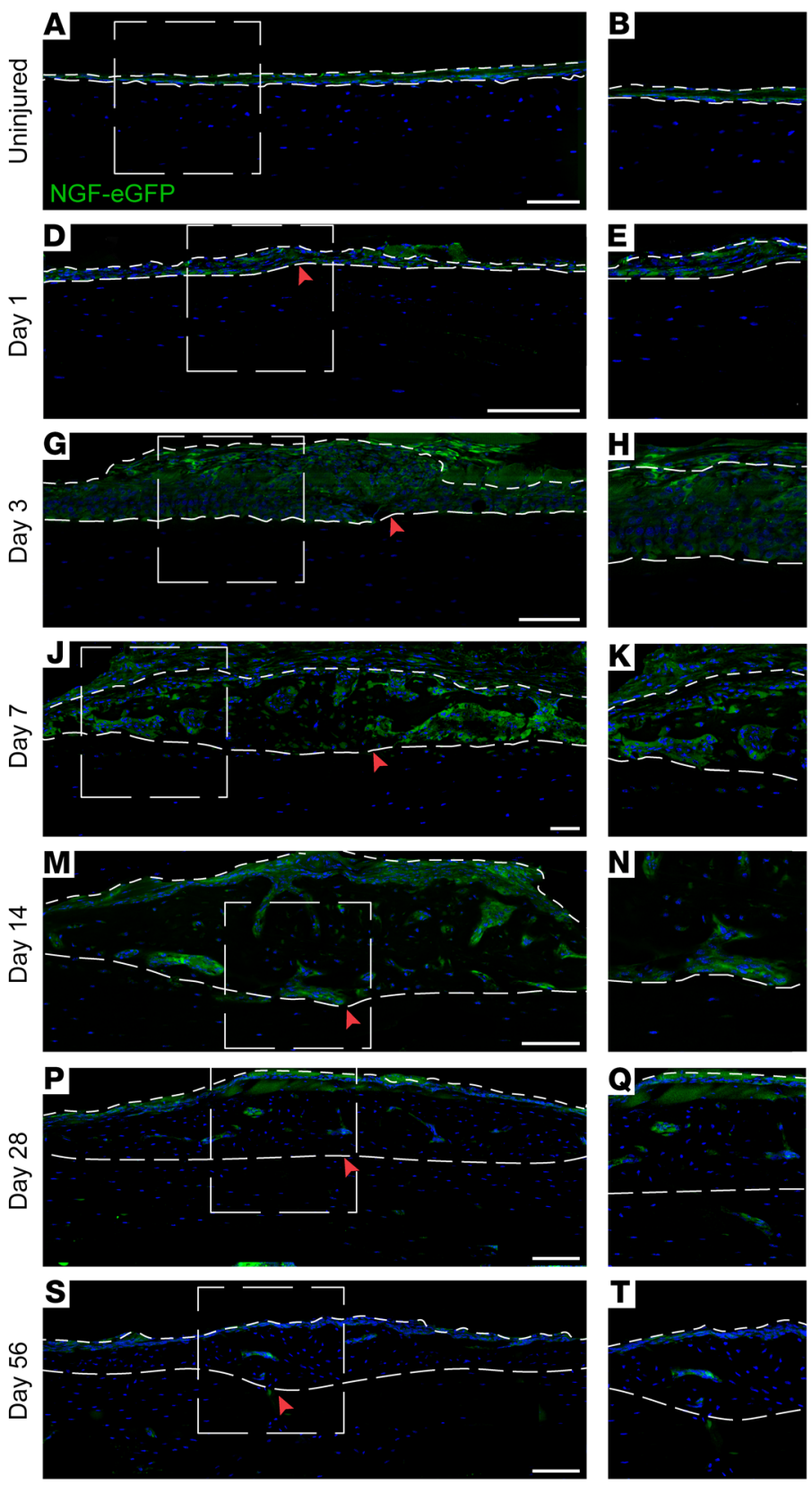
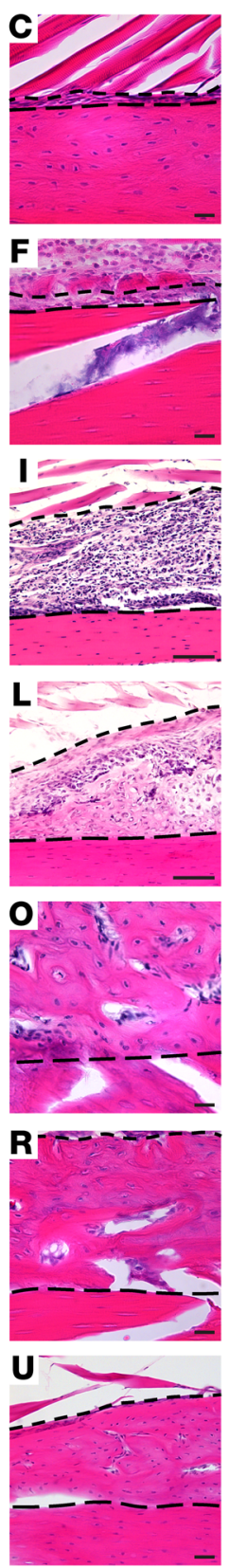

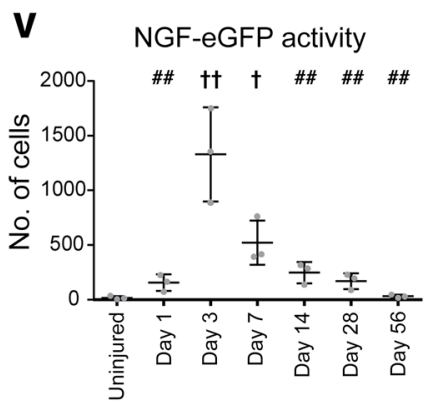

Figure 2. NGF reporter activity after stress fracture. (A-U) Representative tile scans (left), high-magnification images (middle), and representative $\mathrm{H} \& \mathrm{E}$-stained images (right) of the ulnar fracture site and associated callus in NGF-eGFP reporter animals at serial time points between days 1 and 56 after stress fracture. Reporter activity is shown in green, and nuclear counterstaining is shown in blue. An uninjured control is shown for comparison. The thin dashed white line indicates the uppermost boundary of the periosteum or fracture callus. The thicker dashed white line represents the boundary between the periosteum or fracture callus and the underlying cortical bone. Red arrowheads indicate the fracture site. (V) Semiquantitation of NGF-eGFP reporter activity after fracture on days 1-56 in comparison with the uninjured control. Each dot in the graphs represents a single sample, with the sample numbers indicated below. White scale bars: $50 \mu \mathrm{m}$; black scale bars: $20 \mu \mathrm{m}$. Data are expressed as the mean $\pm \mathrm{SD} .{ }^{\dagger} P<0.05$ and ${ }^{\dagger \dagger} P<0.01$ versus the uninjured control; $\# P<0.01$ versus the day-3 time point, by 1-way ANOVA with post hoc Newman-Keuls test.
$5, A-G)$. Similar to periosteal innervation, fine-caliber vessels coursed along the uninjured periosteum and were often seen in a longitudinal fashion in close relationship to nerve fibers (Figure 5A). At early time points preceding bone matrix deposition, vascularity was increased, but this increase in vascularity was often eclipsed by changes in nerve fiber frequency (Figure 5, B and C). In contrast, at time points during bone matrix deposition, callus vascularization became much more well defined and prominent and far exceeded callus innervation (Figure 5, D and E). CD $31^{+}$ vascular channels were ubiquitously present within the interstices of the hard callus, whereas neural elements were again primarily confined to the outer periphery of the fracture callus. At later time points during progressive callus ossification, CD $31^{+}$vessels became less conspicuous (Figure 5, F and G). Next, we quantified $\mathrm{CD} 31^{+}$vessel frequency within the organizing fracture callus over a 56-day post-injury period (Figure $5 \mathrm{H}$ ). We found that CD $31^{+}$ vascularity associated with the periosteal fracture was increased at all time points after injury and had the highest quantitative value on day 7 after fracture, a time point after nerve ingrowth (days 1-3) and before the height of callus ossification (day 14). In sum, both innervation and vascularization of a stress fracture follow a predictable and temporospatial pattern of reaction to injury. Moreover, increases in fracture innervation precede fracture vascularization, ossification, and mineralization (see Figure 5I for a schematic representation).

Disruption of TrkA signaling attenuates reinnervation by sensory nerves and impairs fracture healing. In order to inhibit TrkA, we used a chemical-genetic approach, in which TrkA signaling could be acutely disrupted over defined windows of time. TrkA ${ }^{\mathrm{F} 592 \mathrm{~A}}$ mice are homozygous for knockin alleles that encode a phenylal- 

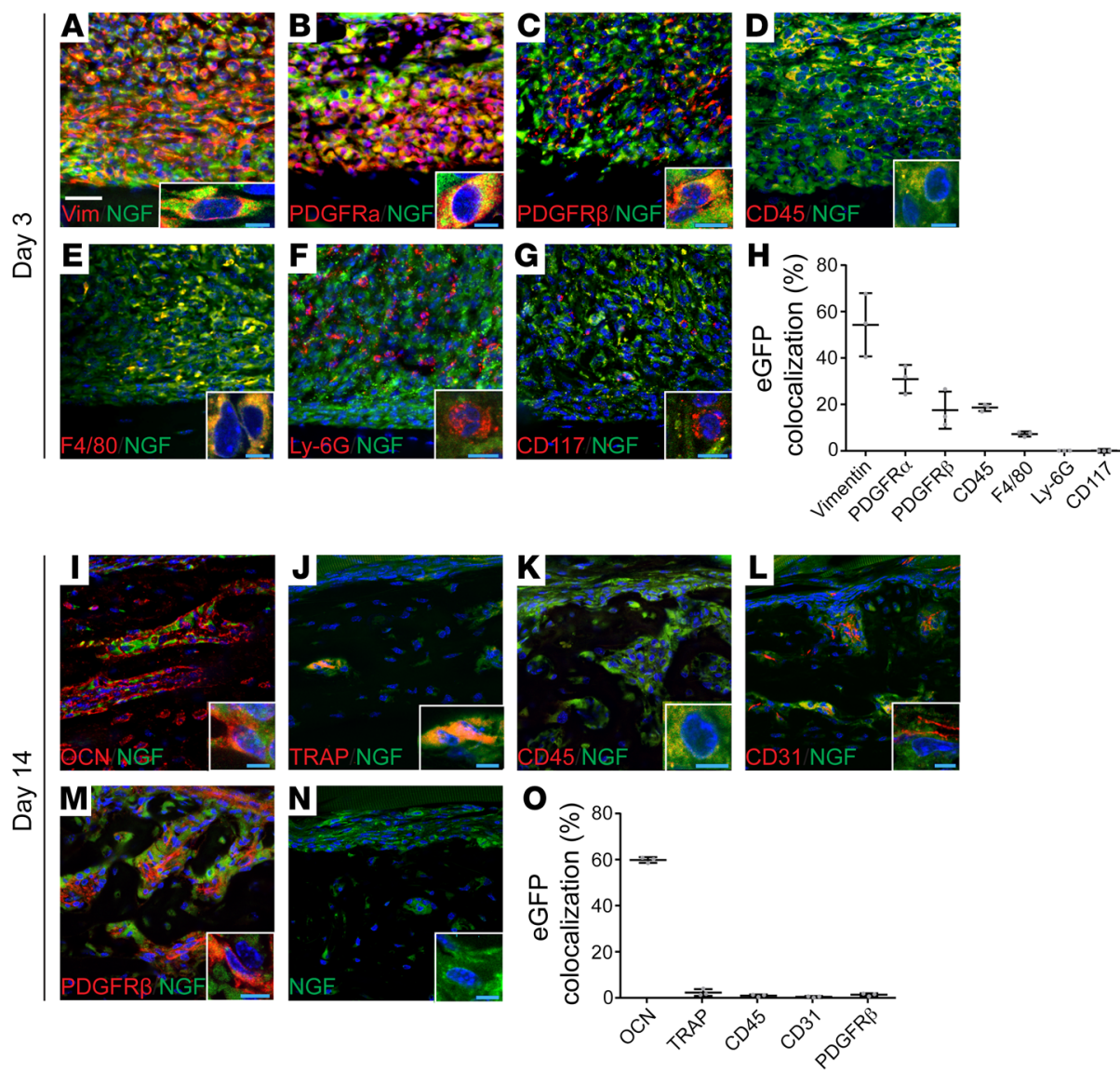

Figure 3. Cellular sources of NGF after stress fracture. (A-C) IHC was performed on a NGF-eGFP fracture callus on day 3 after injury, including staining for (A) vimentin (Vim), (B) PDGFR $\alpha$, (C) PDGFRß, (D) CD45, (E) F4/80, (F) Ly-6G, and (C) CD117. Immunohistochemical staining is shown in red or yellow, and NGF reporter activity is shown in green. Nuclear counterstaining is shown in blue. (H) Semiquantitative analysis of eGFP coexpression with immunofluorescence staining of NGF-eGFP reporter sections on day 3 after fracture. (I-N) Immunohistochemical analysis of a NGF-eGFP fracture callus on day 14 , including staining for (I) osteocalcin (OCN), (J) TRAP, (K) CD45, (L) CD31, (M) PDGFRß, and $(\mathbf{N})$ a negative control without a primary antibody. Immunohistochemical staining is shown in red, NGF reporter activity is shown in green, and nuclear counterstaining is shown in blue. (0) Semiquantitative analysis of eGFP coexpression with immunofluorescence staining of NGF-eGFP reporter tissue sections on day 14 after fracture. In the graphs, each dot represents a single analyzed image. Data are expressed as the mean \pm SD. White scale bar: $50 \mu \mathrm{m}$; blue scale bars (insets): $5 \mu \mathrm{m}$. anine-to-alanine substitution in the protein kinase subdomain $\mathrm{V}$, rendering its catalytic activity sensitive to specific inhibition by the membrane-permeable, small molecule 1NMPP1 (22). To validate this approach, we performed 2 different studies. First, we demonstrated that in vitro exposure to 1NMPP1 prevented dendrite sprouting in dorsal root ganglia (DRGs) isolated from TrkA ${ }^{\mathrm{F} 592 \mathrm{~A}}$ animals (Supplemental Figure 2). Second, in vivo administration of 1NMPP1 to TrkA ${ }^{\mathrm{F} 92 \mathrm{~A}}$ mice caused a marked reduction in TrkA phosphorylation as assessed by immunoblotting of DRG-derived protein isolates (Supplemental Figure 3).

$\mathrm{TrkA}^{\mathrm{F} 592 \mathrm{~A}}$ mice were next subjected to cyclic end-loadinginduced stress fracture with or without 1NMPP1-mediated inhibition of TrkA (Figure 6). For this purpose, all TrkA ${ }^{\text {F592A }}$ mice were crossbred with pan-neuronal reporter Thy1-YFP mice in order to visualize nerve fibers within callus tissue. Animals were sacrificed for analysis on days 7 and 14 after injury - the 2 time points at which the majority of bone is formed within the healing fracture. Innervation, vascularization, and ossification within the ulnar callus tissue were assessed. $\mu \mathrm{CT}$ imaging revealed a reduction in periosteal reactive bone among 1NMPP1-treated animals on day 7 (Figure 6, A-C) and day 14 after injury (Figure 6, D-F). Quantitative $\mu \mathrm{CT}$ metrics of bone formation showed a $51.2 \%$ and $21.0 \%$ reduction in bone volume among the 1NMPP1-treated groups on days 7 and 14 after injury, respectively (Figure 6G). Likewise, we observed a $54.3 \%$ and $22.3 \%$ reduction in callus tissue volume on days 7 and 14, respectively (Figure 6H), whereas callus BMD was similar across treatment groups (Figure 6I). In contrast, $\mu \mathrm{CT}$ analysis of uninjured forelimbs had no detectable change among
1NMPP1-treated TrkA ${ }^{\mathrm{F} 592 \mathrm{~A}}$ animals (Supplemental Figure 4). Moreover, and as further validation, 1NMPP1 showed no detectable effect in stress fracture healing among mice with a WT TrkA allele (Supplemental Figure 5). We next assessed the frequency of Thy $1^{+}$dendrites within the callus of control- and 1NMPP1-treated TrkA $^{\mathrm{F5} 52 \mathrm{~A}}$ Thy1-YFP animals (Figure 6, J-N). $\mathrm{YFP}^{+}$nerve fibers were again noted most prominently on the outer bounds of the hard callus on days 7 and 14 after injury (Figure 6, J and L). We observed a dramatic reduction in $\mathrm{YFP}^{+}$nerve fibers among the 1NMPP1-treated animals (Figure 6, $\mathrm{K}$ and $\mathrm{M}$ ), with a $66.7 \%$ and $78.2 \%$ reduction on days 7 and 14, respectively (Figure $6 \mathrm{~N}$ ). We next assessed callus vascularity among control- and $1 \mathrm{NMPP1}$-treated TrkA ${ }^{\mathrm{F} 592 \mathrm{~A}}$ animals (Figure 6, O-S). Control-treated mice again showed a large number of $\mathrm{CD} 1^{+}$vessels within the interstices between bone trabeculae of the hard callus (Figure 6, O and Q). We detected a reduction in $\mathrm{CD} 31^{+}$vascular channels within 1NMPP1-treated fracture sites (Figure 6, P and R), which, after quantification, showed a $29.3 \%$ and $18.5 \%$ reduction on post-injury days 7 and 14 , respectively (Figure 6S). Alkaline phosphatase (ALP) staining of cross sections of the fracture sites confirmed a significant reduction in osteoblastic activity in 1NMPP1-treated TrkA ${ }^{\mathrm{F} 592 \mathrm{~A}}$ animals (Figure 6, T and U), reflecting a $42.4 \%$ reduction in ALP staining intensity on post-injury day 7 (Figure 6V). In aggregate, inhibition of TrkA catalytic activity within skeletal sensory nerves over the time course of stress fracture led to marked reductions in fracture-associated innervation, blunted revascularization, reduced total osteoblastic activity, and a substantial delay in ossification of the fracture callus. 

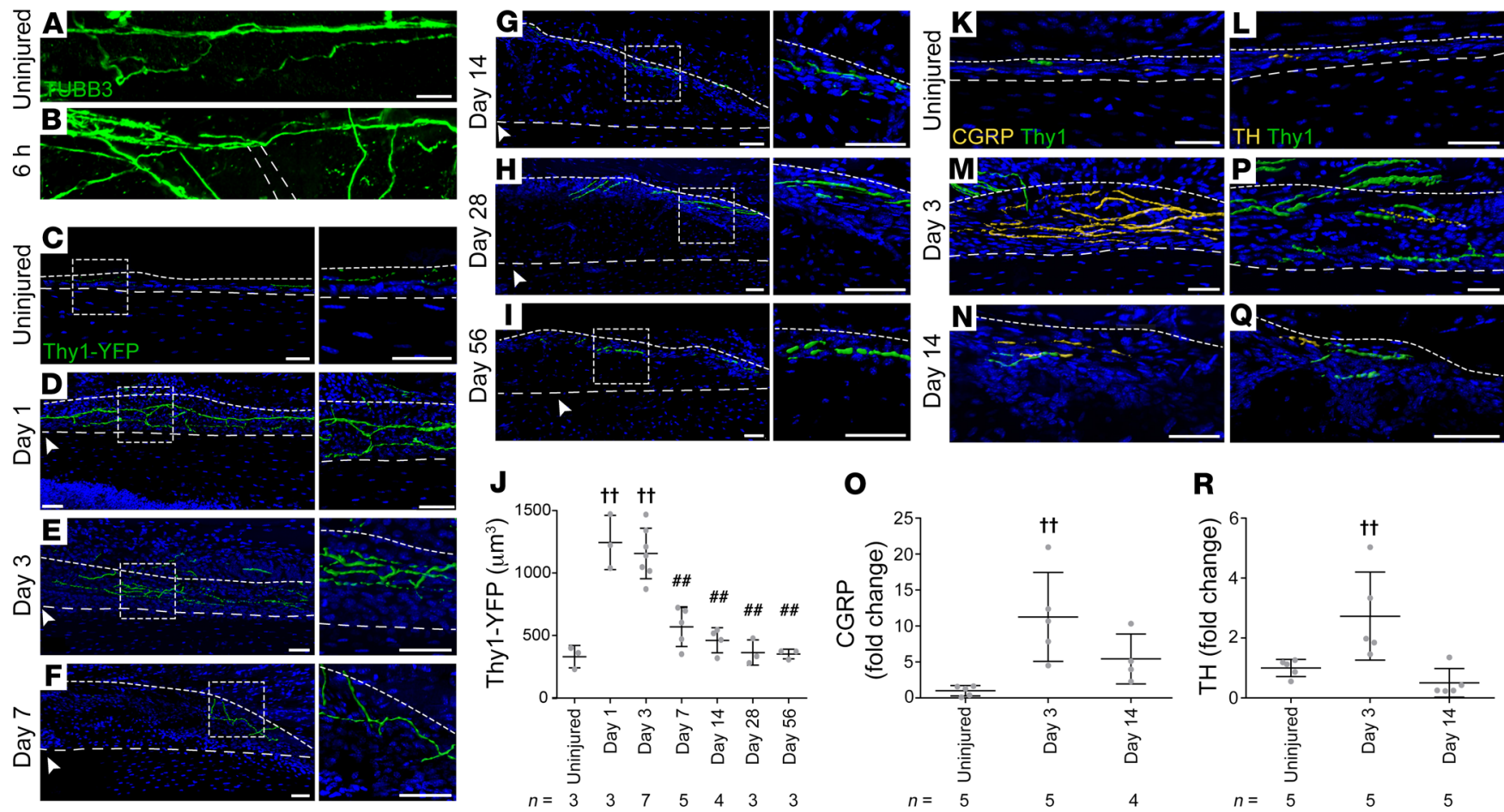

0

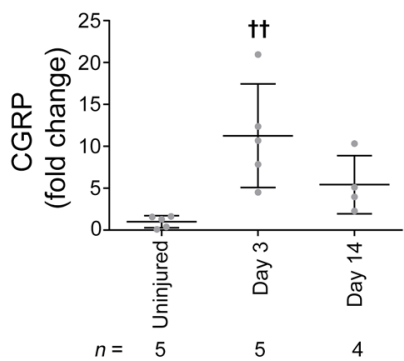

$\mathbf{R}$

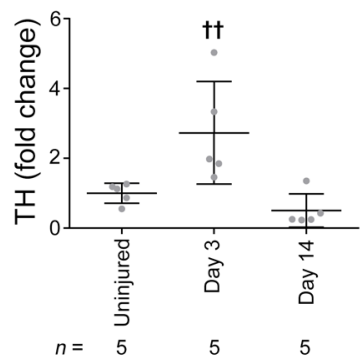

Figure 4. TrkA+CGRP+ sensory nerve sprouting after stress fracture. (A and B) TUBB3 whole-mount immunohistochemical staining of the ulnar periosteum before and 6 hours after stress fracture. White dashed lines indicate the fracture site. (C-I) Tile scans and high-magnification images of ulnar fracture calluses in Thy1-YFP reporter animals, with reporter activity shown in green using Imaris surface renderings. (C) Uninjured control. (D-I) Fracture callus on days $3,7,14,28$, and 56 after injury. The thin dashed white line represents the uppermost boundary of the periosteum or fracture callus. The thick dashed white line represents the boundary between the periosteum or fracture callus and the underlying cortical bone. White arrowheads indicate the fracture site. (J) Quantification of Thy1-YFP reporter activity from days 1-56 after fracture, indicated as a volumetric measure of YFP reporter activity. (K and $\mathbf{L}$ ) Representative images of CGRP and TH immunohistochemical detection (yellow) in uninjured periosteum from Thy1-YFP reporter (green) animals. (M and $\mathbf{N}$ ) CGRP immunohistochemical detection (yellow) and ( $\mathbf{0}$ ) quantification on days 3 and 14 after fracture. (P and $\mathbf{Q}$ ) TH immunohistochemical detection (yellow) and (R) quantification ons day 3 and 14 after fracture. In the graphs, each dot represents a single sample, with the sample numbers indicated below. Scale bars: $50 \mu \mathrm{m}$ and $20 \mu \mathrm{m}$ (insets). Data are expressed as the mean \pm SD. ${ }^{\dagger+} P<0.01$ versus the uninjured control; ${ }^{\# \#} P<0.01$ versus the day-1 time point, by 1-way ANOVA with post hoc Newman-Keuls test.

PTX-induced peripheral neuropathy impairs fracture healing. Chemotherapeutic agents often induce a length-dependent peripheral neuropathy, and distal sensory nerve fibers are particularly sensitive to microtubule stabilization via taxanes $(23,24)$. We hypothesized that sensory polyneuropathy in WT mice would phenocopy the blunted fracture repair process previously observed in mice with conditional TrkA alleles.

As previously observed $(23,24)$, low-dose PTX induced characteristics of a length-dependent sensory neuropathy (Supplemental Figure 6). This included a $34.2 \%$ reduction in PGP9.5 intraepidermal nerve fibers (Supplemental Figure 6, A-C), a corresponding $34.5 \%$ reduction in $\mathrm{CGRP}^{+}$fibers within the paw skin (Supplemental Figure 6, D-F), as well as thermal hypoalgesia (Supplemental Figure 6G). Small nerve fiber frequency within the uninjured ulnar periosteum was similarly reduced by $44.2 \%$ (Figure $7, \mathrm{~A}-\mathrm{C}$ ), but no measurable change in cortical bone parameters was observed within the uninjured ulna by $\mu \mathrm{CT}$ imaging or by quantitative analysis (Supplemental Figure 7).

Next, and to assess the effects of PTX-induced sensory neuropathy on fracture healing, we subjected WT mice to ulnar stress fracture after PTX treatment. By post-injury days 7 and 14, PTX-treated mice showed a clear reduction in callus size by $\mu \mathrm{CT}$ reconstruction and cross-sectional imaging across both time points (Figure 7, D-I). Quantitative $\mu \mathrm{CT}$ analysis revealed a significant reduction in $\mathrm{BV}$ (43.3\% and $60.2 \%$ reduction on days 7 and 14 ) and BMD (7.5\% and 9.1\% reduction on days 7 and 14) (Figure 7, J and K). Nerve sprouting within the callus tissue was substantially reduced, as assessed by $\mathrm{TUBB}^{+}$nerve fibers (Figure 7, $\mathrm{L}$ and $\mathrm{M}$; day 7 results are shown). Quantification revealed a $53.7 \%$ and $44.7 \%$ reduction in nerve fiber frequency within the callus tissue among PTX-treated animals on days 7 and 14 after stress fracture, respectively (Figure 7N). Callus vascularity, as assessed by CD31 immunostaining, showed a concomitant $44.1 \%$ and $39.6 \%$ reduction on days 7 and 14 after injury, respectively (Figure 7, O-Q). Thus, sensory neuropathy induced by PTX recapitulated key features of TrkA inhibition, including reduced callus innervation, vascularization, and osteoblast activity within the stress fracture site.

\section{Discussion}

In this study, we sought to define the cellular events that accompany the reinnervation of the fracture callus in genetic mouse models that enabled sequential, specific, and quantitative assessment of components of sensory nerve NGF/TrkA signaling. We show that NGF is acutely upregulated following stress fracture, triggering 

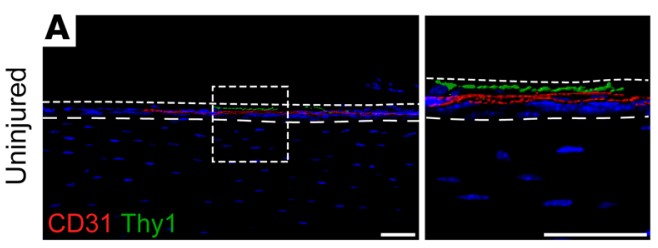

\section{H}
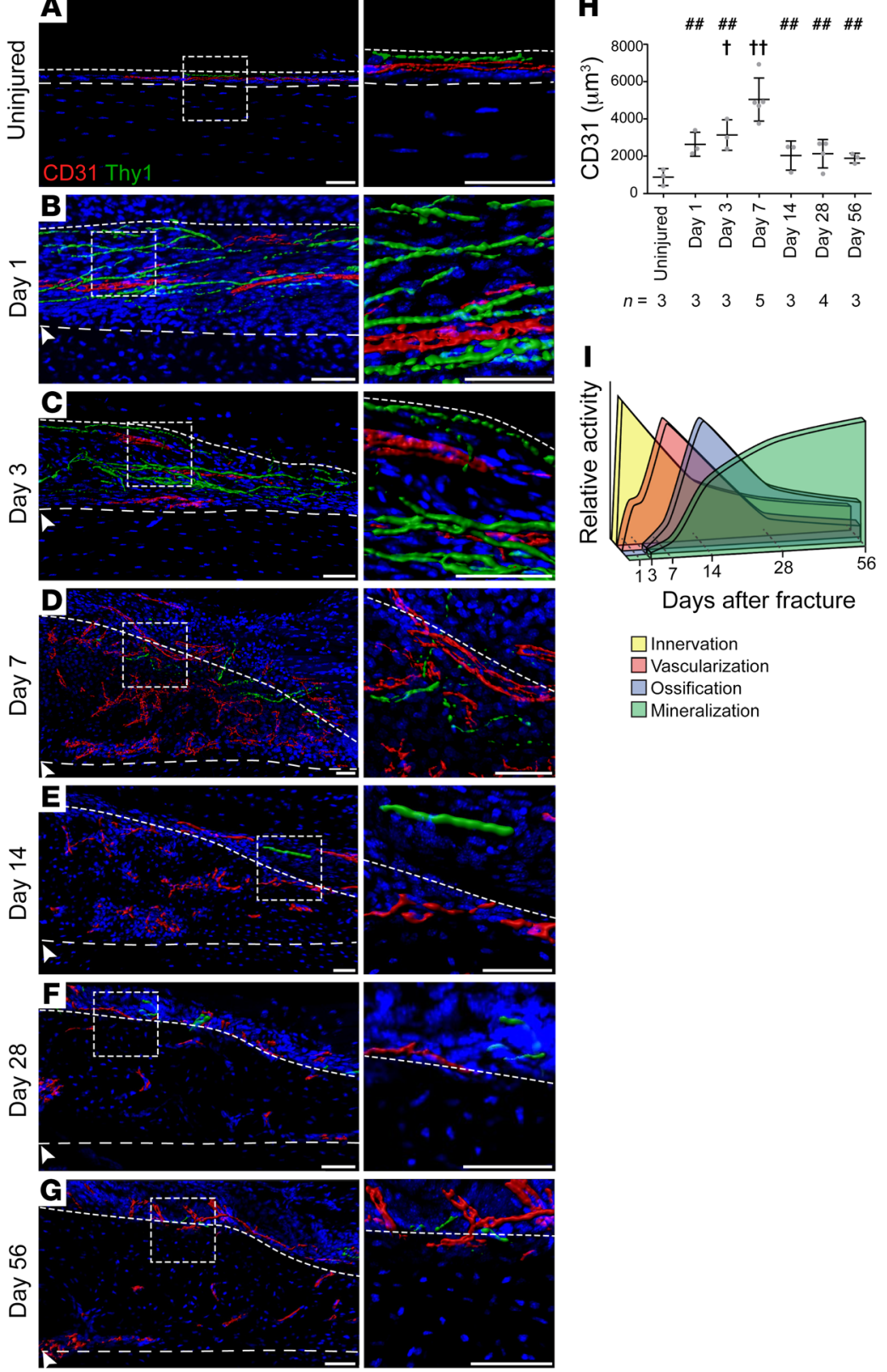

Figure 5. Neurovascular changes after stress fracture.

(A-C) Representative CD31 immunohistochemical staining and quantification after fracture, performed on Thy1-YFP reporter fracture sites. Imaris surface renderings of CD31 immunohistochemical staining (red) and Thy1-YFP reporter activity (green), with nuclear counterstaining shown in blue. Tile scans of longitudinal cross sections of the fracture callus are shown along with high-magnification images. (A) Uninjured control. (B-C) Fracture callus on days $3,7,14,28$, and 56 after injury. The thin dashed white line indicates the uppermost boundary of the periosteum or fracture callus. The thick dashed white line represents the boundary between the periosteum or fracture callus and the underlying cortical bone. White arrowheads indicate the fracture site. (H) Quantification of CD31 immunohistochemical staining at serial time points from days 1-56 after fracture, reported as a volumetric measure of CD31 immunohistochemical staining. (I) Schematic model of the temporal sequence of events after ulnar stress fracture. The number of days after fracture is depicted on the $x$ axis, and the relative activity of each cellular process is shown on the $y$ axis. In the graphs, each dot represents a single sample, with the number of samples indicated below. Scale bars: $50 \mu \mathrm{m}$ (including insets). Data are expressed as the mean $\pm \mathrm{SD} .{ }^{\dagger} P<0.05$ and ${ }^{+t} P<0.01$ versus the uninjured control; ${ }^{\# P} P<0.01$ versus the day-7 time point, by 1-way ANOVA with post hoc Newman-Keuls test.

activity impairs a sequence of events in long bone ossification, including impeding the expansion of osterix-expressing progenitor cell numbers and blunting vascular ingrowth (10). Given our data demonstrating the critical role of TrkA signaling in bone morphogenesis and repair, it is not clear why experimental work with neutralizing antibodies against NGF have not shown a significant delay in bone healing $(28,29)$. It is possible that the analgesic effects of anti-NGF are observed at a much lower dose than the effects needed to impede tissue repair. The extent to which these pro-regenerative properties of TrkA signaling can be harnessed to drive repair of the mammalian skeleton is not yet known. Certainly, studies in amphibians suggest this possi-

TrkA signaling by sensory nerves, which is required for reinnervation, vascularization, and subsequent osteoblast synthetic activity within the fracture callus. An independent method to induce skeletal sensory neuropathy by PTX treatment phenocopied these observations. These findings are generally compatible with the neurotropic model operating during organogenesis, in which tissues dictate the amount and type of innervation they require by secreting NGF, which promotes neuronal survival by retrograde activation of TrkA.

The requirement for skeletal sensory nerves for mammalian bone tissue repair is also consistent with findings from studies in lower-order species. These studies showed that appendage regeneration in starfish, certain amphibians, and fish is a nerve-dependent process (25-27). Moreover, our previous studies in mouse long bone development showed that interruption of perichondrium-derived NGF expression or sensory nerve-associated TrkA bility, in which, for example, surgical rerouting of nerve branches in axolotls can prompt the formation of an accessory limb (30).

An important question that remains to be addressed involves identifying the molecular mechanisms that mediate neuroskeletal intercommunication, and in particular the secondary messengers elaborated by sensory nerve fibers responsible for positive regulation of osteoblastogenesis. Results in amphibian models suggest that nerve-derived factors including FGFs $(31,32)$, anterior gradient protein $(26)$, and BMPs $(31,32)$ can promote limb regeneration. Sonic hedgehog (Shh) has been implicated as a nerve-derived signal in Xenopus limb regeneration (33) as well as in the regulation of progenitor cells within the murine hair follicle (34) or incisor (35). Wnt ligands have been shown to be sequestered in nerve axons (36), and alterations in Wnt signaling activity are associated with an impaired regenerative response in denervated mouse digit tips (37). In our prior observations, the activation of sensory neurons 
Day 7
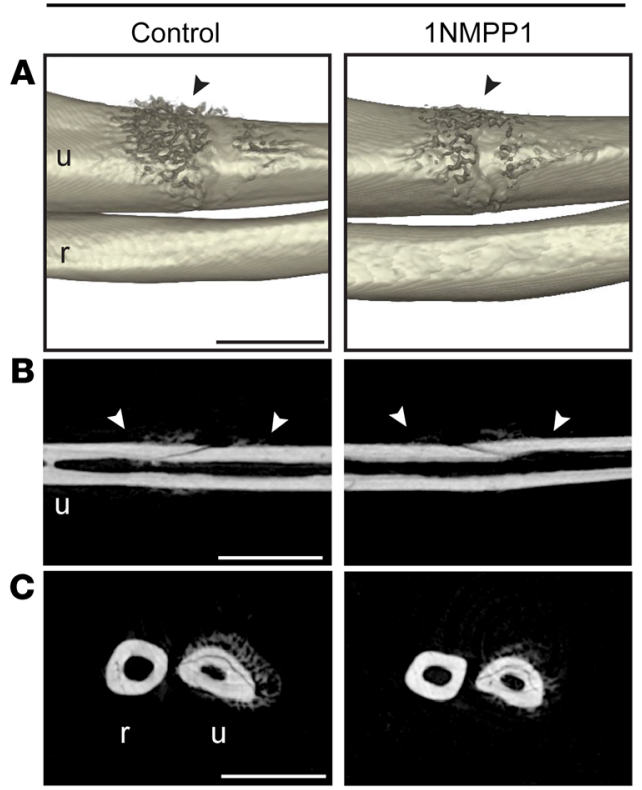

Day 14

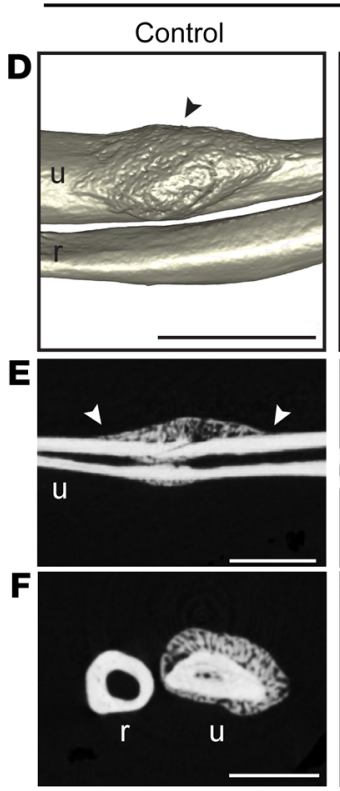

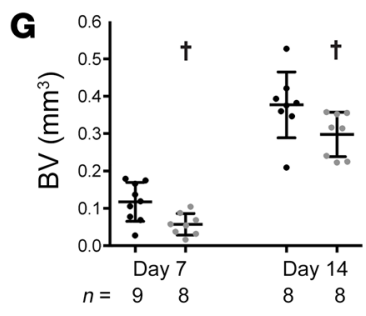
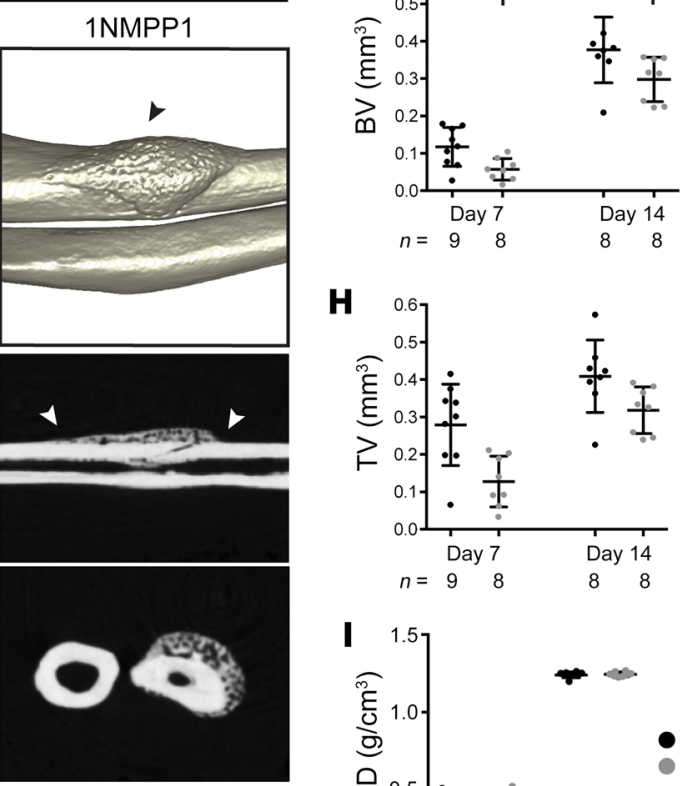

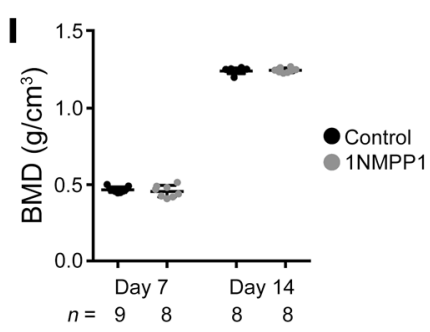

Thy1-YFP
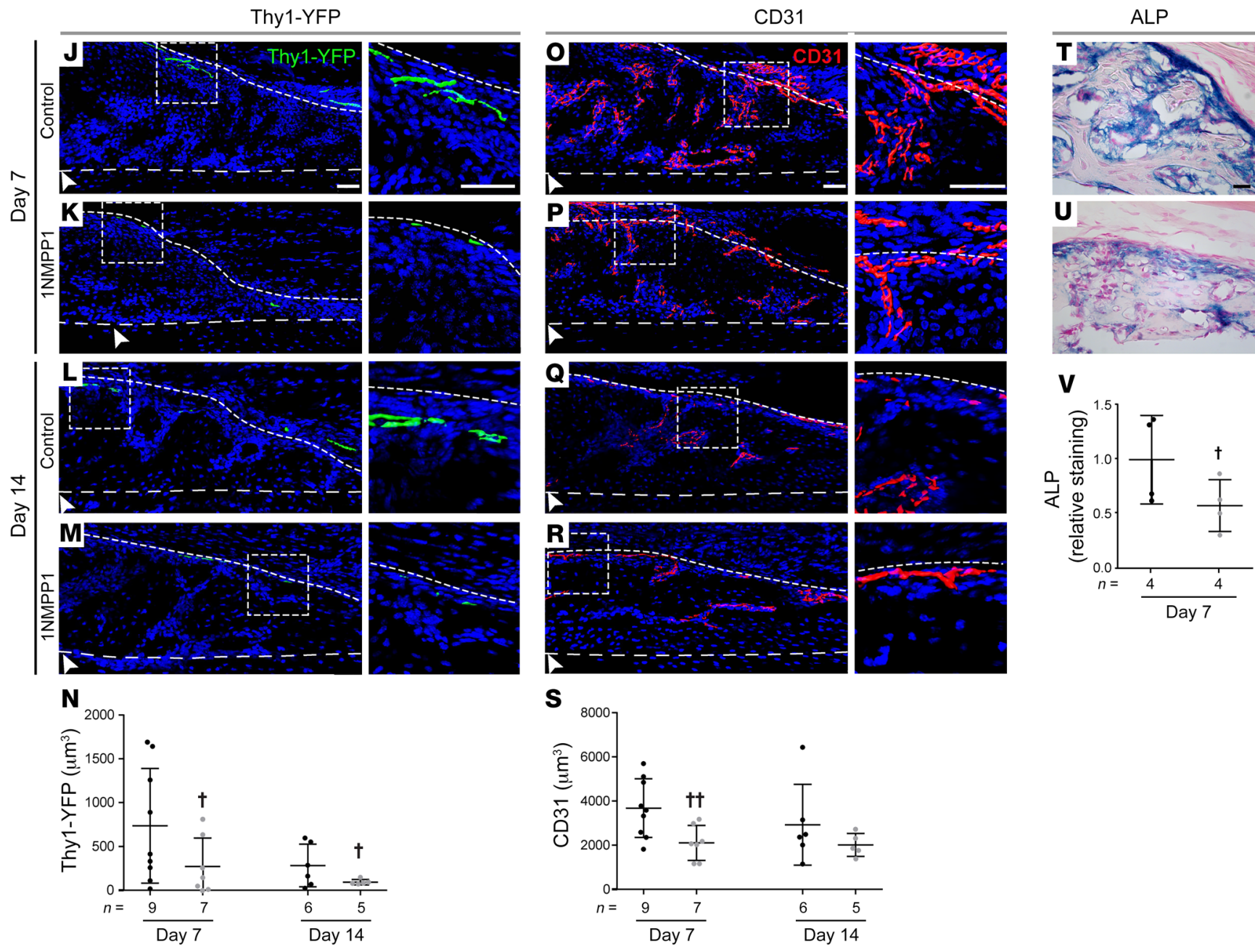
Figure 6. Inhibition of TrkA catalytic activity and its effects on stress fracture healing. (A-F) $\mu \mathrm{CT}$ images of ulnar stress fracture healing in TrkA ${ }^{\text {F592A }}$ Thy1-YFP mice treated with 1NMPP1 or vehicle control on (A-C) day 7 and (D-F) day 14 after injury. (A) $\mu$ CT reconstructions, (B) coronal cross-sectional images, and (C) axial cross-sectional images. (G-I) Quantitative $\mu C T$ analysis of stress fractures in TrkA ${ }^{\mathrm{F} 592 \mathrm{~A}}$ Thy1-YFP mice on days 7 and 14 after injury. (G) BV, (H) TV, and (I) BMD of callus. (J-M) Thy1-YFP reporter activity in TrkA ${ }^{\text {F592A }}$ Thy1-YFP mice treated with (J and $\mathbf{L}$ ) vehicle control or (K and $\mathbf{M}$ ) 1NMPP1, as seen on day 7 after injury. Tile scans and high-magnification images are shown. The thin dashed white line indicates the uppermost boundary of the fracture callus. The thick dashed white line represents the boundary between the fracture callus and the underlying cortex. White arrowheads indicate the fracture site. (N) Quantification of Thy1-YFP reporter activity on days 7 and 14 after injury. (O-R) CD31 immunohistochemical staining of tissue from TrkA ${ }^{\text {F592A }}$ Thy1-YFP mice treated with ( $\mathbf{O}$ and $\mathbf{Q}$ ) vehicle control or ( $\mathbf{P}$ and $\mathbf{R}$ ) 1NMPP1, on day 7 after injury. Tile scans and high-magnification images are shown. (S) Quantification of CD31 immunohistochemical staining, 7 and 14 days after injury. (T and $\mathbf{U}$ ) High-magnification images of ALP staining of tissue from TrkA ${ }^{\mathrm{F} 592 \mathrm{~A}}$ Thy1-YFP mice treated with (T) vehicle control or (U) 1NMPP1, on day 7 after injury. (V) Quantification of ALP staining intensity, on day 7 after injury. For all graphs, each dot represents a single sample, with sample numbers indicated below. Scale bars: $500 \mu \mathrm{m}$ (A and D), $1 \mathrm{~mm}$ (B, C, E, and F), $50 \mu \mathrm{m}$ (J-R), $25 \mu \mathrm{m}$ (enlarged insets in $\mathbf{A}-\mathbf{R}, \mathbf{T}$ and $\mathbf{U}$ ). Data are expressed as the mean $\pm \mathrm{SD} .{ }^{\dagger} P<0.05$ versus the vehicle control at the corresponding time point, by 2-tailed Student's $t$ test.

was necessary for load-induced osteocytic Wnt signaling activity (38). Other groups have focused on the role of the classic neuropeptides substance P and CGRP, found in skeletal sensory dendrites, and their direct effects on osteoblasts (38-43). Nevertheless, the physiologically relevant secondary signals released from TrkAexpressing nerve endings during stress fracture remain unknown and are the subject of continued investigation.

The phenotypic similarities of deficient nerve regrowth and attenuated fracture healing seen in PTX-treated and TrkAmutant mice suggest a common pathophysiologic mechanism for defective tissue repair in states of peripheral neuropathy. PTX is extensively used in the treatment of solid tumors (44), and taxane-induced peripheral neuropathy is a frequent and dose-limiting complication $(45,46)$. Chemotherapy-induced peripheral neuropathy (CIPN) is seen in up to $80 \%$ to $90 \%$ of patients (47), and sensory nerves in which their cell bodies are at a great distance from terminal dendrites are particularly sensitive to microtubular stabilization. Thus, PTX has been shown to induce distal axonal degeneration, a reduction in intraepidermal nerve frequency, and thermal hypoalgesia characteristic of sensory neuropathy $(23,24)$. PTX has been shown to impair nerve growth after experimental injury, accompanied by reduced NGF at the injury site (48). In clinical scenarios, chemotherapeutic agents are well known to reduce bone mineral density in patients, even after stratification for hormonal changes or disease stage (49). In addition, several recent studies of distraction osteogenesis in dogs demonstrated that the neurotoxic agent cisplatin inhibits distraction-induced osseous repair $(50,51)$. It is intriguing to speculate on the extent to which neuropathy in other disease states, such as diabetes, has a contributory role in poor bone repair.

Several important limitations exist with regard to a broader extrapolation of the findings from the present study. First, the model used is an experimental stress fracture one that minimal- ly disrupts the skeletal tissue, including bone-associated nerve fibers. It is not yet known whether NGF/TrkA signaling modulates bone repair in larger or more extensive bone injuries in an analogous fashion. For example, in a displaced fracture or segmental bone defect model, small nerve fibers would probably be transected, and the requirement for reinnervation of nerve fibers to meet their target tissue would be higher. Second, as a note on our experimental design, throughout the study the contralateral limb was used as an uninjured control. We cannot exclude the possibility that stress fracture in 1 forelimb could result in changes to the contralateral limb that were not overtly clear.

Several additional lines of circumstantial evidence support the relevance of our findings in humans. First, neutralizing NGF antibodies have been found to predispose individuals to an unusually high rate of subchondral insufficiency fractures that come to clinical attention (52). Second, missense and nonsense mutations in TRKA (NTRK1) cause congenital insensitivity to pain with anhidrosis (CIPA) syndrome (53), which is characterized in part by skeletal dysplasia and delayed fracture healing $(54,55)$. A similar human phenotype is observed in patients with homozygous mutations in NGF that result in impaired processing of pro-NGF (56, 57). Finally, common peripheral neuropathies, such as diabetic neuropathy, have well-known associations with delayed fracture repair. In fact, the presence of neuropathy in patients with diabetes is an independent risk factor for failed fracture repair (58). These lines of evidence in patients suggest that the influence of sensory nerves on bone repair under normal or pathologic conditions may well be an overlooked facet of human skeletal biology.

\section{Methods}

Mouse models. Details of the mouse lines used are summarized in Supplemental Table 1. NGF-eGFP mice, which express eGFP under the control of the mouse NGF promoter, were donated by the Kawaja laboratory (16). Thy1-YFP mice, which harbor a transgene derived from the mouse Thy1 gene that directs expression of YFP in motor and sensory neurons, are commercially available (17). TrkA ${ }^{+}$nerve fibers were visualized using TrkA-LacZ mice, which have a LacZ sequence inserted immediately downstream of the ATG in exon 1 of the murine Ntrk1 gene (18). TrkA ${ }^{\mathrm{F} 592 \mathrm{~A}}$ mice are homozygous for a phenylalanine-to-alanine point mutation in exon 12 of the mouse Ntrk1 gene (F592A), rendering the endogenous TrkA kinase sensitive to inhibition by the membrane-permeable, small molecule 1NMPP1 (22). For all experiments, TrkA ${ }^{\mathrm{F} 592 \mathrm{~A}}$ mice were crossed with Thy1-YFP reporter mice (referred to as TrkA ${ }^{\mathrm{F} 592 \mathrm{~A}}$ Thy1-YFP mice). When feasible, littermate analysis was performed by investigators blinded to the mouse genotype.

In order to achieve temporal inhibition of TrkA catalytic activity in TrkA ${ }^{\mathrm{F} 92 \mathrm{~A}}$ animals, the small molecule $1 \mathrm{NMPP} 1$ was used, which was synthesized by Aurora Analytics LLC using standard techniques (59). Purity (99.2\%) was confirmed by HPLC-UV254, and characterization by ${ }^{1} \mathrm{H}$ NMR (400 MHz, DMSO-d6) was consistent with structure. Stock solution was prepared at $200 \mathrm{mM}$ by dissolving 1 NMPP1 powder in DMSO. For 1NMPP1 administration, i.p. injections were given 48 hours before and 2 hours before loading using a $5-\mathrm{mM}$ solution at a dosage of $17 \mu \mathrm{g} / \mathrm{g}$ BW. DMSO containing vehicle was used for control treatment. Animals were thereafter maintained on 1NMPP1-treated drinking water ( $40 \mu \mathrm{M} 1 \mathrm{NMPP} 1$ in $\mathrm{dd}_{2} \mathrm{O}$ with $1 \%$ PBS-Tween 20 ). 

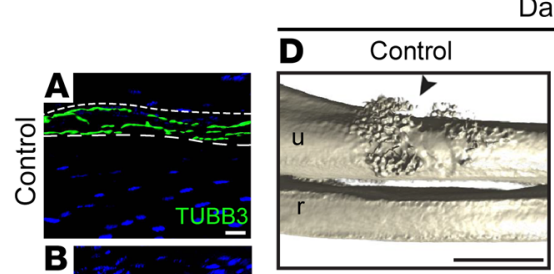

Day 7
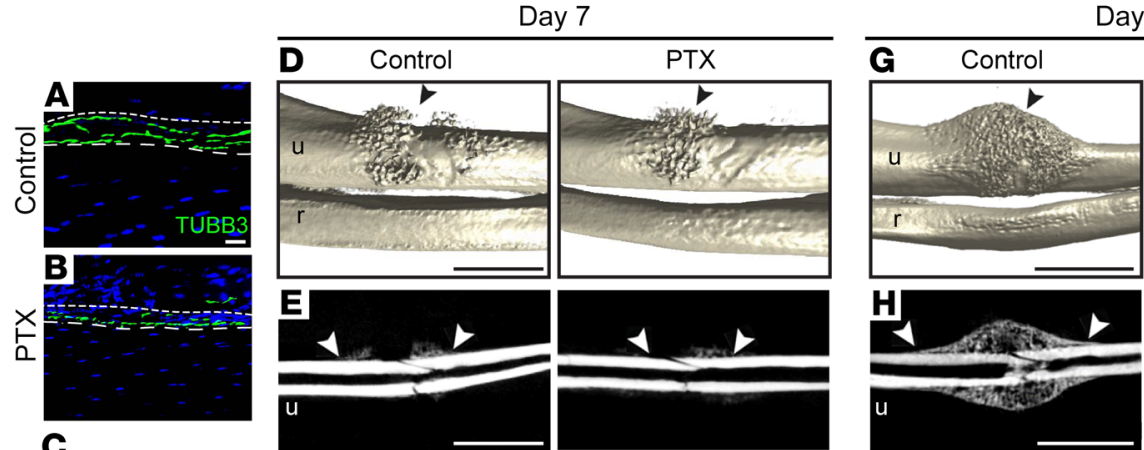

Day 14

$$
\text { C }
$$
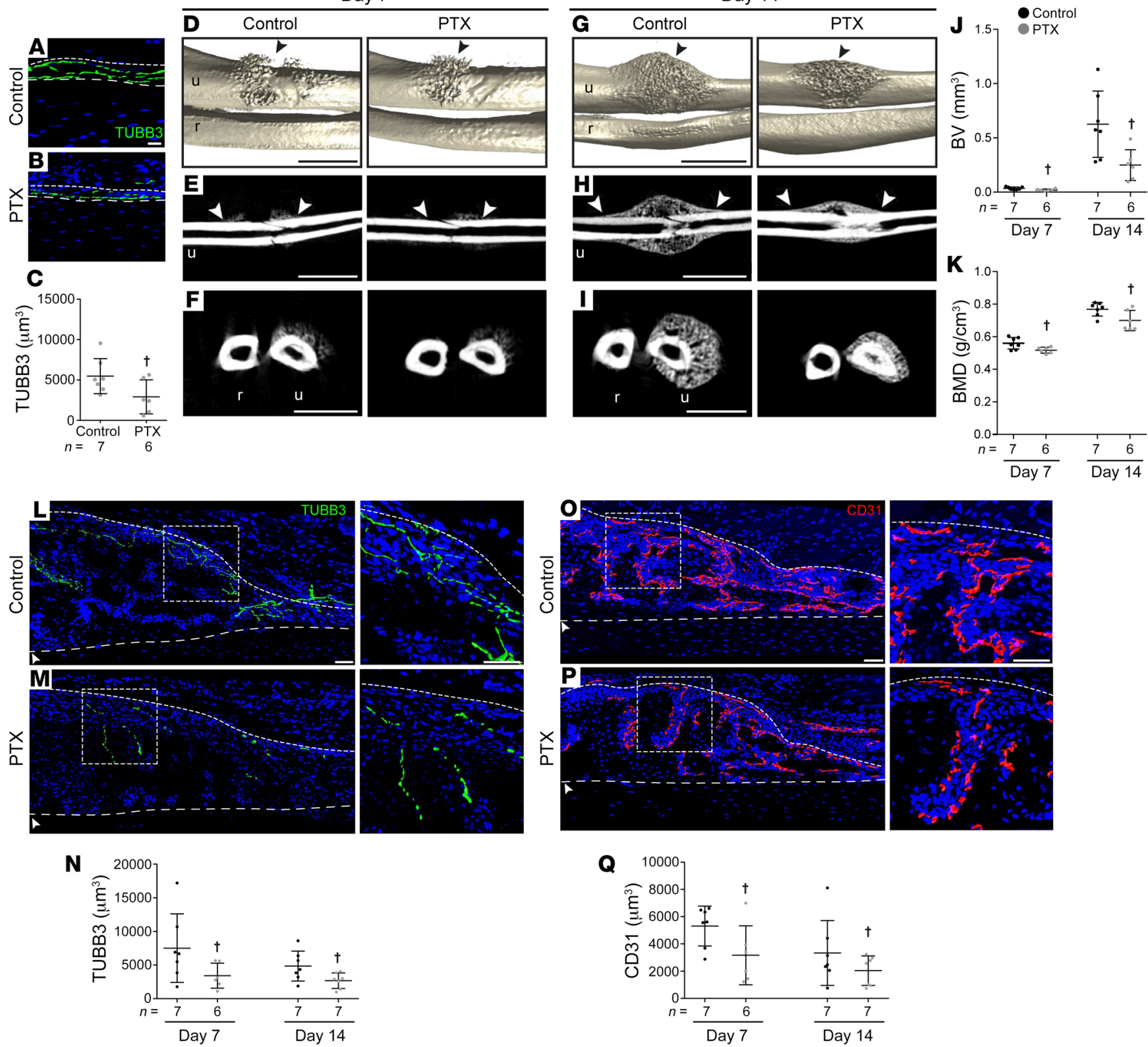

Figure 7. Chemotherapy-induced peripheral neuropathy phenocopies TrkA inhibition during stress fracture. (A-C) Periosteal nerve fiber loss in animals treated with PTX within the uninjured ulnar periosteum. (A and B) Representative images of TUBB3 immunohistochemical staining (green) and (C) quantification of TUBB3 ${ }^{+}$nerve fiber frequency. (D-I) $\mu$ CT images of ulnar stress fracture healing in control- or PTX-treated mice on day 7 after injury. (D and $\left.\mathbf{G}\right)$ $\mu \mathrm{CT}$ reconstructions of the fracture site, $(\mathbf{E}$ and $\mathbf{H})$ coronal cross-sectional images, and (F and $\mathbf{I})$ axial cross-sectional images on days 7 and 14 after injury. ( and $\mathbf{K}$ ) Quantitative $\mu \mathrm{CT}$ analysis of control- or PTX-treated mice on days 7 and 14 after injury. (J) BV of callus and (K) BMD of callus. (L and M) Immunohistochemical staining for TUBB3+ nerve fibers among (L) control- or (M) PTX-treated mice on day 7 after injury. Tile scans and high-magnification images are shown. The thin dashed white line indicates the uppermost boundary of the fracture callus, and the thick dashed white line indicates the boundary between the fracture callus and the underlying cortex. White arrowheads indicate the fracture site. (N) Quantification of TUBB3 immunohistochemical staining on days 7 and 14 after injury. ( $\mathbf{O}$ and $\mathbf{P})$ CD31 immunohistochemical staining of tissue from (0) control- or (P) PTX-treated animals on day 7 after injury. Tile scans and high-magnification images are shown. (Q) Quantification of CD31 immunohistochemical staining of tissue from control- or PTX-treated mice on days 7 and 14 after injury. Each dot represents a single sample, with sample numbers indicated below. Scale bars: $50 \mu \mathrm{m}(\mathbf{A}, \mathbf{B}, \mathbf{L}, \mathbf{M}, \mathbf{0}$, and $\mathbf{P}$ and insets in $\mathbf{L}$, $\mathbf{M}, \mathbf{O}$, and $\mathbf{P}), 1 \mathrm{~mm}\left(\mathbf{E}, \mathbf{F}, \mathbf{H}\right.$, and $\mathbf{I}$ ), and $500 \mu \mathrm{m}$ ( $\mathbf{D}$ and $\mathbf{G}$ ). Data are expressed as the mean $\pm \mathrm{SD} .{ }^{\dagger} P<0.05$ versus control, by 2-tailed Student's $t$ test.

Induction of stress fracture. Cyclic end-loading of the ulna was performed in adult male mice (18 weeks of age) as adapted from previously validated protocols (60). Mice were anesthetized with isoflurane gas (1\%-3\% inhaled) for the duration of the experiment. After confirming deep anesthesia, the right forelimb was axially compressed by placing the olecranon process and the flexed carpus into specially designed fixtures. A material testing system (TA Instruments, ElectroForce 3200 Series II) was used to apply force and monitor displacement. A compressive loading force was applied with a peak force $(\mathrm{F})$ as determined individually for each mouse line. Loading was termi- 
nated when a displacement constant (D) was reached. High-resolution Faxitron imaging was used to identify displaced fractures or combined ulnar/radial fractures, which occurred in a minority of mice that were hence euthanized and excluded from analysis (Supplemental Figure 8; Faxitron Bioptics). Left forelimbs were not loaded and were used as an uninjured control. After fracture, mice were given an i.p. injection of analgesic $(0.05 \mathrm{mg} / \mathrm{kg}$ buprenorphine) and allowed unrestricted cage activity. Bilateral ulnas were harvested 0-56 days after loading.

In our hands, as observed in prior studies (60), variation between mouse lines was present in the force and displacement required for induction of stress fracture. For this reason and for each mouse line, 3-4 males of the same age were used for calibration to determine the ultimate force (F) and displacement constant (D). Briefly, the left forelimb was first loaded monotonically to determine ultimate force, and the right forelimb was next fatigue-loaded to determine displacement to fracture. Monotonic loading was performed by a displacement ramp of $0.5 \mathrm{~mm} / \mathrm{s}$ to complete the displaced fracture, and the average ultimate force was recorded for each animal line (Supplemental Table 2). The contralateral forelimb was next fatigue-loaded at $75 \%$ of the average ultimate force, and displacement data were analyzed to determine peak (maximum) actuator displacement for each cycle, and from these values we determined the increase in peak actuator displacement from cycle 10 to complete displaced fracture. As in prior studies, cycle 10 was used as a reference, because there is substantial soft tissue preconditioning during the first few cycles (60). The average increase in peak displacement to complete fracture for each animal line was recorded. For survival fatigue loading, $66.67 \%$ of the peak displacement to fracture was used as the displacement constant (D).

Chemotherapy-induced sensory neuropathy. Peripheral sensory neuropathy induced by PTX was performed using previously reported methods $(23,24)$. Male 15.5-week-old C57BL/6J mice received PTX (Taxol, $25 \mathrm{mg} / \mathrm{kg}$ by tail vein injection, every 48 hours for a total of 3 doses; Sigma-Aldrich, T7402). Thermal sensation was assessed using the IITC Plantar Analgesia Meter 400 (IITC Life Science). Paw withdrawal latency was performed before and after treatment according to the Hargreaves method (24). Two weeks after the final dose (at 18 weeks of age), cyclic end-loading was performed as outlined above to induce a stress fracture. Animals were sacrificed 7 and 14 days thereafter, and bilateral forelimbs were isolated and analyzed as outlined below. In addition, intraepidermal nerve fiber loss was confirmed in a medial footpad biopsy from the left hind limb on the basis of previously reported methods $(23,24)$. Briefly, $50-\mu \mathrm{m}$-thick cryosections of the skin biopsy site were analyzed by immunohistochemical staining for the pan-axonal marker protein gene product (PGP) 9.5 (Bio-Rad) or calcitonin gene-related peptide (CGRP).

$\mu C T$ imaging and analysis. Forelimbs were dissected free of skin and evaluated using a SkyScan 1172 high-resolution $\mu \mathrm{CT}$ imaging system (Bruker). Each forelimb was scanned separately at $65 \mathrm{kV}$ and 153 $\mu \mathrm{A}$ with a $1.0-\mathrm{mm}$ aluminum filter to obtain a $9-\mu \mathrm{m}$ voxel size. Scan slices were acquired in the transverse plane by placing the bone parallel to the $z$ axis of the scanner. NRecon (Bruker) was used to reconstruct images with the following settings: ring artifact reduction of $5 \%$ and beam-hardening correction of $20 \%$, and quantitative analysis was performed using CTAn (Bruker) in accordance with the recommendations of the American Society for Bone and Mineral Research (61). Volumes of interest (VOI) encompassed the entire callus and excluded native bony elements. All analyses were performed in a blinded fashion.
Histology and IHC. Forelimbs were harvested and placed in $4 \%$ paraformaldehyde (PFA) at $4^{\circ} \mathrm{C}$ for 24 hours. After 3 washes in PBS, samples were decalcified in 14\% EDTA (1:20 volume, Sigma-Aldrich) for up to 28 days at $4^{\circ} \mathrm{C}$. Samples were either processed for paraffin embedment (10- $\mu$ m-thick sections) or cryosection (10- or 50- $\mu$ m-thick sections). For cryosections, samples were cryoprotected in $30 \%$ sucrose overnight at $4^{\circ} \mathrm{C}$ before embedment in OCT (Tissue-Tek, 4583). Longitudinal sections were mounted on adhesive slides (TruBond 380). H\&E staining was performed using standard protocols. ALP staining was performed using prepared reagents, according to the manufacturer's instructions (Sigma-Aldrich, 85L2-1KT). For IHC analysis, sections were washed 3 times in PBS for 5 minutes. For immunostaining of thick $(50-\mu \mathrm{m})$ sections (used for tyrosine hydroxylase [TH], CGRP, TUBB3, PGP9.5, and CD31 immunostaining), the sections were first permeabilized with $0.5 \%$ Triton-X for 30 minutes prior to blocking. All slides were blocked with $5 \%$ normal goat serum for 30 minutes and then incubated in primary antibodies overnight at $4^{\circ} \mathrm{C}$ in a humidified chamber (see Supplemental Table 3 for a complete list of the antibodies used). The following day, slides were washed in PBS, incubated in the appropriate fluorescent secondary antibody, A594 goat anti-rabbit or A647 goat anti-mouse, for 1 hour at $25^{\circ} \mathrm{C}$, and then mounted with Vectashield HardSet Antifade Mounting Medium with DAPI (Vector Laboratories, Vectashield H-1500). Digital images of these sections were captured with $\times 10$ to $\times 100$ objectives using upright fluorescence microscopy (Leica Microsystems, Leica DM6) or confocal microscopy (Carl Zeiss Microscopy, Zeiss LSM780 FCS).

Whole-mount IHC. Injured forelimbs from C57BL/6J mice were used for whole-mount immunohistochemical staining to visualize nerve fibers. Prior to immunostaining, the skin and muscle were carefully dissected away from the ulna under a dissecting microscope, exposing the periosteum. The samples were fixed in $4 \%$ PFA at $4^{\circ} \mathrm{C}$ overnight. Samples were then washed with PBS 3 times and permeabilized with $0.5 \%$ Triton X-100 at room temperature for 30 minutes. To block nonspecific binding, samples were submerged in $1 \mathrm{~mL} \mathrm{10 \%}$ normal goat serum at room temperature for 30 minutes. The primary antibody was then applied ( $1 \mathrm{~mL}$ anti-TUBB3, 1:1500) at $4^{\circ} \mathrm{C}$ overnight. Samples were then washed 3 times in PBS and submerged in the appropriate secondary antibody $(1 \mathrm{~mL}$ A594 goat anti-rabbit IgG, 1:200) for 1 hour at room temperature. Next, ulnas were washed 3 times in PBS and submerged in PBS in a black PELCO dish (Ted Pella Inc.). Imaging was performed using the $\times 20$ water immersion lens and 2-photon excitation on a Zeiss 710 NLO Axio Examiner Multiphoton Upright Confocal Microscope.

$X$-Gal staining. Cryosections of TrkA-LacZ forelimbs were washed in PBS for 10 minutes. Samples were washed (wash buffer: $200 \mu \mathrm{L}$ of $1 \mathrm{M} \mathrm{MgCl}, 10 \mathrm{mg}$ sodium deoxycholate, $0.02 \% \mathrm{~Np}-40$ [Triton X-100] in $100 \mathrm{~mL}$ of $0.1 \mathrm{M}$ PBS) for 15 minutes 3 times. Samples were placed in fresh X-gal staining solution ( $200 \mu \mathrm{L} \mathrm{X-gal} \mathrm{stock,} 100 \mu \mathrm{L}$ of $0.5 \mathrm{M}$ potassium ferricyanide, $100 \mu \mathrm{L}$ of $0.5 \mathrm{M}$ potassium ferrocyanide, $200 \mu \mathrm{L}$ of $1 \mathrm{M}$ Tris, $9.4 \mathrm{~mL}$ wash buffer) for 96 hours at $37^{\circ} \mathrm{C}$. Samples were then washed twice in wash buffer for 15 minutes and counterstained with Nuclear Fast Red (Sigma-Aldrich, N8002) for 5 minutes, placed under running tap water for 1 minute, dehydrated, and mounted using permanent mounting medium. The specificity of staining was confirmed using the fracture sites from age-matched animals not expressing LacZ.

Histologic image analysis. All images for quantification were obtained using either an upright fluorescence microscope (Leica 
Microsystems, Leica DM6) or a confocal microscope (Carl Zeiss Microscopy, Zeiss LSM780 FCS). NGF-eGFP reporter activity was quantified by manually counting the entire number of eGFP ${ }^{+}$cells in longitudinal cross sections of the callus for each animal. TrkA-LacZ reporter activity and ALP staining were quantified with the magic wand tool in Photoshop CC 2017 (Adobe) with a tolerance of 30, using random $\times 40$ microscopic fields within the callus tissue. Prior studies have suggested that anti-GFP antibodies may be required for complete detection of small nerve fibers in Thy1-YFP reporter animals (62). For this reason, we examined reporter activity with or without anti-GFP staining (Supplemental Figure 9). Here, fracture sites from Thy1-YFP reporter cryosections were stained with anti-GFP-A488 and imaged on a Zeiss LSM-780-FCS confocal microscope with an EC Plan-Neofluar 10x.0.03 M27 objective lens using the 514 laser at a power of 4.0. In order to perform spectral unmixing of the endogenous yellow fluorescent protein (YFP) fluorescence and the anti-GFP-A488, a lambda scan was then performed between 508 and $695 \mathrm{~nm}$, with images acquired every $8.9 \mathrm{~nm}$.

Thy1-YFP reporter activity was quantified with the surfacerendering function in Imaris on the green channel. $\mathrm{YFP}^{+}$surfaces were identified with the following settings: smooth surface detail of $1 \mu \mathrm{m}$, background subtraction with a diameter of the largest sphere of $3 \mu \mathrm{m}$, and seed points of $4 \mu \mathrm{m}$ in a $100-\mu \mathrm{m}^{2}$ region of interest (ROI). Each $100-\mu \mathrm{m}^{2}$ ROI was captured at $200-\mu \mathrm{m}$ intervals along the fracture callus, and data are presented as the mean per $100-\mu \mathrm{m}^{2}$ ROI across each sample. Likewise, CD31, TH, and CGRP immunohistochemical stainings were quantified using the surface-rendering function of Imaris on the red channel for $\mathrm{CD} 31^{+}$blood vessels, $\mathrm{TH}^{+}$ nerves, and $\mathrm{CGRP}^{+}$nerves using an analogous protocol and with the following settings: smooth surface detail of $1 \mu \mathrm{m}$, background subtraction with a diameter of the largest sphere of $3 \mu \mathrm{m}$, and seed points of $4 \mu \mathrm{m}$ in a $100-\mu \mathrm{m}^{2}$ ROI.

Dissociated DRG neuronal culture. For the dissociated DRG neuronal culture, DRGs were harvested from TrkA ${ }^{\mathrm{F} 592 \mathrm{~A}}$ at E13.5 and transferred into ice-cold culture medium containing DMEM/F-12 supplemented with 5\% FBS (Gibco, Thermo Fisher Scientific) and 1\% penicillin/streptomycin (Life Technologies, Thermo Fisher Scientific). Next, DRGs were digested with $1 \mathrm{mg} / \mathrm{mL}$ collagenase A (Roche, 10103578001) at $37^{\circ} \mathrm{C}$ for 15 minutes, and then with $0.05 \%$ trypsinEDTA at $37^{\circ} \mathrm{C}$ for 7 minutes. DRGs were then washed 3 times with culture medium and dissociated by trituration with a $1-\mathrm{mL}$ pipette tip. The dissociated neurons were plated into poly-D-lysine/laminincoated 12-well plates at a density of $10^{4}$ neurons $/ \mathrm{cm}^{2}$. Non-neuronal cells were eliminated using $20 \mu \mathrm{M} 5$-fluoro-2-deoxyuridine and $20 \mu \mathrm{M}$ uridine (FDU/R). NGF (50 ng/mL, Gibco, Thermo Fisher Scientific, 13257-019) was added to each well except for the negative control. The treated groups received different concentrations of 1NMPP1 for the entire culturing course. DRG neurons were cultured for 96 hours followed by visualization with an anti-neurofilament antibody (Thermo Fisher Scientific, MA110041).

Western blot analysis. Four pairs of DRGs (L2-L5) were harvested from 16- to 18-week-old male TrkA ${ }^{\mathrm{F} 592 \mathrm{~A}}$ mice who had received either 1NMPP1 or vehicle control treatment. Microdissected DRGs were placed into ice-cold Triton X-100 lysis buffer containing both a protease inhibitor (Sigma-Aldrich, P8340) and a phosphatase inhibitor (Sigma-Aldrich, P2850). DRGs were then minced in the ice-cold lysis buffer followed by incubation for 5 minutes on ice and centrif- ugation for 10 minutes at 12,000 rpm for protein extraction. TrkA phosphorylation was assessed by Western blotting using a p-TrkA antibody in comparison with the $\beta$-actin loading control (Cell Signaling Technology).

Statistics. Quantitative data are expressed as the mean \pm SD, with $P$ values of less than 0.05 and 0.01 considered significant. Individual data points are shown, and the number of samples or images analyzed are indicated in the figures and/or legends. A Shapiro-Wilk test for normality was performed on all data sets. Homogeneity was confirmed by a comparison of variances test. Parametric data were analyzed using the appropriate Student's $t$ test when 2 groups were compared, or a 1-way ANOVA when more than 2 groups were compared, followed by a post hoc Newman-Keuls test to compare 2 groups. Sample size calculations were performed for experiments presented in Figure 6 on the basis of an anticipated effect size of 1.75 , using our previously published data in adult TrkA ${ }^{\mathrm{F} 92 \mathrm{~A}}$ mice (38). For this scenario, with 8 replicates per group, a 2 -sample $t$ test would provide $80 \%$ power to detect effect sizes of at least 1.5, assuming a 2-sided 0.05 level of significance. In vitro experiments were performed in technical and biological triplicate unless otherwise described.

Study approval. All animals were housed and procedures performed under a protocol approved by the IACUC of Johns Hopkins University (protocol M015M118).

\section{Author contributions}

AWJ and TLC conceived and designed the study, acquired funding, and approved the final manuscript. Zhu Li, CAM, LC, Zhi Li, SL, and RT acquired, analyzed, and interpreted data. Zhu Li, CAM, LC, AH, AWJ, and TLC prepared the manuscript. Zhi Li performed all in vivo experiments and radiographic imaging, and CAM and LC performed all postmortem analyses. The order of the co-first authors was determined by weighing the scope and depth of the contribution of each person and was agreed on by all authors.

\section{Acknowledgments}

AWJ was supported by the National Institute of Arthritis and Musculoskeletal and Skin Diseases (NIAMS), NIH (R01 AR070773, K08 AR068316); the National Institute of Dental and Craniofacial Research (NIDCR), NIH (R21 DE027922); the Department of Defense (USAMRAA W81XWH-18-1-0336, W81XWH-18-1-0121, W81XWH-18-10613); the American Cancer Society (Research Scholar Grant RSG-18-027-01-CSM); the Maryland Stem Cell Research Foundation; and the Musculoskeletal Transplant Foundation. TLC was supported by the NIAMS, NIH (R01 AR068934); the NIDCR, NIH (R21 DE027922); and the Veterans Administration (VA) (Merit Award and Senior Research Career Scientist Award). The content is solely the responsibility of the authors and does not necessarily represent the official views of the NIH or the Department of Defense. We thank the Johns Hopkins University microscopy facility and Sarah Miller and Qizhi Qin for their technical assistance.

Address correspondence to: Thomas L. Clemens, 601 North Caroline Street, Suite 5242, Baltimore, Maryland 21287, USA. Phone: 410.955.3245; Email: tclemen5@jhmi.edu. Or to: Aaron W. James, 720 Rutland Avenue, Room 524A, Baltimore, Maryland 21205, USA. Phone: 410.502.4143; Email: awjames@jhmi.edu. 
1. Bjurholm A, Kreicbergs A, Brodin E, Schultzberg M. Substance P- and CGRP-immunoreactive nerves in bone. Peptides. 1988;9(1):165-171.

2. Castañeda-Corral G, et al. The majority of myelinated and unmyelinated sensory nerve fibers that innervate bone express the tropomyosin receptor kinase A. Neuroscience. 2011;178:196-207.

3. Pezet S, McMahon SB. Neurotrophins: mediators and modulators of pain. Annu Rev Neurosci. 2006;29:507-538.

4. Mantyh PW. The neurobiology of skeletal pain. Eur J Neurosci. 2014;39(3):508-519.

5. Kumar A, Brockes JP. Nerve dependence in tissue, organ, and appendage regeneration. Trends Neurosci. 2012;35(11):691-699.

6. Reichardt LF. Neurotrophin-regulated signalling pathways. Philos Trans R Soc Lond, B, Biol Sci. 2006;361(1473):1545-1564.

7. Huang EJ, Reichardt LF. Trk receptors: roles in neuronal signal transduction. Annu Rev Biochem. 2003;72:609-642.

8. Kaplan DR, Stephens RM. Neurotrophin signal transduction by the Trk receptor. J Neurobiol. 1994;25(11):1404-1417.

9. Howe CL, Valletta JS, Rusnak AS, Mobley WC. NGF signaling from clathrin-coated vesicles: evidence that signaling endosomes serve as a platform for the Ras-MAPK pathway. Neuron. 2001;32(5):801-814.

10. Tomlinson RE, et al. NGF-TrkA signaling by sensory nerves coordinates the vascularization and ossification of developing endochondral bone. Cell Rep. 2016;16(10):2723-2735.

11. Grills BL, Schuijers JA. Immunohistochemical localization of nerve growth factor in fractured and unfractured rat bone. Acta Orthop Scand. 1998;69(4):415-419.

12. Asaumi K, Nakanishi T, Asahara H, Inoue H, Takigawa M. Expression of neurotrophins and their receptors (TRK) during fracture healing. Bone. 2000;26(6):625-633.

13. Hukkanen M, et al. Rapid proliferation of calcitonin gene-related peptide-immunoreactive nerves during healing of rat tibial fracture suggests neural involvement in bone growth and remodelling. Neuroscience. 1993;54(4):969-979.

14.Madsen JE, et al. Fracture healing and callus innervation after peripheral nerve resection in rats. Clin Orthop Relat Res. 1998;(351):230-240.

15. Nordsletten L, et al. The neuronal regulation of fracture healing. Effects of sciatic nerve resection in rat tibia. Acta Orthop Scand. 1994;65(3):299-304.

16. Kawaja MD, et al. Nerve growth factor promoter activity revealed in mice expressing enhanced green fluorescent protein. JComp Neurol. 2011;519(13):2522-2545.

17. Feng G, et al. Imaging neuronal subsets in transgenic mice expressing multiple spectral variants of GFP. Neuron. 2000;28(1):41-51.

18. Moqrich A, et al. Expressing TrkC from the TrkA locus causes a subset of dorsal root ganglia neurons to switch fate. Nat Neurosci. 2004;7(8):812-818

19. Bjurholm A, Kreicbergs A, Terenius L, Goldstein M, Schultzberg M. Neuropeptide Y-, tyrosine hydroxylase- and vasoactive intestinal polypeptide- immunoreactive nerves in bone and surrounding tissues. J Auton Nerv Syst. 1988;25(2-3):119-125.

20. Martin P, Lewis J. Origins of the neurovascular bundle: interactions between developing nerves and blood vessels in embryonic chick skin. Int J Dev Biol. 1989;33(3):379-387.

21. Li W, et al. Peripheral nerve-derived CXCL12 and VEGF-A regulate the patterning of arterial vessel branching in developing limb skin. Dev Cell. 2013;24(4):359-371.

22. Chen $X$, et al. A chemical-genetic approach to studying neurotrophin signaling. Neuron. 2005;46(1):13-21.

23. Turkiew E, Falconer D, Reed N, Höke A. Deletion of Sarm1 gene is neuroprotective in two models of peripheral neuropathy. J Peripher Nerv Syst. 2017;22(3):162-171.

24. Zhu J, Chen W, Mi R, Zhou C, Reed N, Höke A. Ethoxyquin prevents chemotherapy-induced neurotoxicity via Hsp90 modulation. Ann Neurol. 2013;74(6):893-904.

25. Simões MG, et al. Denervation impairs regeneration of amputated zebrafish fins. BMC Dev Biol. 2014;14:49.

26. Kumar A, Godwin JW, Gates PB, Garza-Garcia AA, Brockes JP. Molecular basis for the nerve dependence of limb regeneration in an adult vertebrate. Science. 2007;318(5851):772-777.

27. Huet M. [Role of the nervous system during the regeneration of an arm in a starfish: Asterina gibbosa Penn. (Echinodermata, Asteriidae)]. JEmbryol Exp Morphol. 1975;33(3):535-552.

28. Koewler NJ, et al. Effects of a monoclonal antibody raised against nerve growth factor on skeletal pain and bone healing after fracture of the C57BL/6J mouse femur. J Bone Miner Res. 2007;22(11):1732-1742.

29. Rapp AE, et al. Analgesia via blockade of NGF/ TrkA signaling does not influence fracture healing in mice. J Orthop Res. 2015;33(8):1235-1241.

30. Maden M, Holder N. Axial characteristics of nerve induced supernumerary limbs in the axolotl. Wilehm Roux Arch Dev Biol. 1984;193(6):394-401.

31. Makanae A, Mitogawa K, Satoh A. Co-operative Bmp- and Fgf-signaling inputs convert skin wound healing to limb formation in urodele amphibians. Dev Biol. 2014;396(1):57-66.

32. Makanae A, Mitogawa K, Satoh A. Cooperative inputs of Bmp and Fgf signaling induce tail regeneration in urodele amphibians. Dev Biol. 2016;410(1):45-55.

33. Mitogawa K, Makanae A, Satoh A. Hyperinnervation improves Xenopus laevis limb regeneration. Dev Biol. 2018;433(2):276-286.

34. Brownell I, Guevara E, Bai CB, Loomis CA, Joyner AL. Nerve-derived sonic hedgehog defines a niche for hair follicle stem cells capable of becoming epidermal stem cells. Cell Stem Cell. 2011;8(5):552-565.

35. Zhao H, et al. Secretion of shh by a neurovascular bundle niche supports mesenchymal stem cell homeostasis in the adult mouse incisor. Cell Stem Cell. 2014;14(2):160-173.

36. Inestrosa NC, Arenas E. Emerging roles of Wnts in the adult nervous system. Nat Rev Neurosci. 2010;11(2):77-86.

37. Takeo $\mathrm{M}$, et al. Wnt activation in nail epithelium couples nail growth to digit regeneration. Nature.
2013;499(7457):228-232.

38. Tomlinson RE, et al. NGF-TrkA signaling in sensory nerves is required for skeletal adaptation to mechanical loads in mice. Proc Natl Acad Sci USA. 2017;114(18):E3632-E3641.

39. Wang L, et al. Substance P stimulates bone marrow stromal cell osteogenic activity, osteoclast differentiation, and resorption activity in vitro. Bone. 2009;45(2):309-320.

40. Lundberg P, et al. Greater bone formation of $\mathrm{Y} 2$ knockout mice is associated with increased osteoprogenitor numbers and altered Y1 receptor expression.J Biol Chem. 2007;282(26):19082-19091.

41. Long H, Ahmed M, Ackermann P, Stark A, Li J. Neuropeptide $Y$ innervation during fracture healing and remodeling. A study of angulated tibial fractures in the rat. Acta Orthop. 2010;81(5):639-646.

42. Sample SJ, et al. Role of calcitonin gene-related peptide in functional adaptation of the skeleton. PLOS ONE. 2014;9(12):e113959.

43. Zhang Y, et al. Implant-derived magnesium induces local neuronal production of CGRP to improve bone-fracture healing in rats. Nat Med . 2016;22(10):1160-1169.

44. Mekhail TM, Markman M. Paclitaxel in cancer therapy. Expert Opin Pharmacother. 2002;3(6):755-766.

45. Song SJ, et al. Incidence of taxane-induced peripheral neuropathy receiving treatment and prescription patterns in patients with breast cancer. Support Care Cancer. 2017;25(7):2241-2248

46. Bao T, Basal C, Seluzicki C, Li SQ, Seidman $\mathrm{AD}$, Mao JJ. Long-term chemotherapy-induced peripheral neuropathy among breast cancer survivors: prevalence, risk factors, and fall risk. Breast Cancer Res Treat. 2016;159(2):327-333.

47. Höke A. Animal models of peripheral neuropathies. Neurotherapeutics. 2012;9(2):262-269.

48. Hsu ST, Yao CH, Hsu YM, Lin JH, Chen YH, Chen YS. Effects of Taxol on regeneration in a rat sciatic nerve transection model. Sci Rep. 2017;7:42280.

49. Cameron DA, Douglas S, Brown JE, Anderson RA. Bone mineral density loss during adjuvant chemotherapy in pre-menopausal women with early breast cancer: is it dependent on oestrogen deficiency? Breast Cancer Res Treat. 2010;123(3):805-814

50. Stine KC, et al. Nutlin-3 treatment spares cisplatin-induced inhibition of bone healing while maintaining osteosarcoma toxicity. JOrthop Res. 2016;34(10):1716-1724.

51. Stine KC, et al. Cisplatin inhibits bone healing during distraction osteogenesis. JOrthop Res. 2014;32(3):464-470.

52. Hochberg MC. Serious joint-related adverse events in randomized controlled trials of antinerve growth factor monoclonal antibodies. Osteoarthr Cartil. 2015;23(Suppl 1):S18-S21.

53. Indo $\mathrm{Y}$, et al. Mutations in the TRKA/NGF receptor gene in patients with congenital insensitivity to pain with anhidrosis. Nat Genet. 1996;13(4):485-488.

54. Toscano E, et al. Multisystem involvement in congenital insensitivity to pain with anhidrosis (CIPA), a nerve growth factor receptor(Trk A)- 
related disorder. Neuropediatrics. 2000;31(1):39-41. 55. Bonkowsky JL, Johnson J, Carey JC, Smith AG, Swoboda KJ. An infant with primary tooth loss and palmar hyperkeratosis: a novel mutation in the NTRK1 gene causing congenital insensitivity to pain with anhidrosis. Pediatrics. 2003;112(3 Pt 1):e237-e241.

56. Carvalho OP, et al. A novel NGF mutation clarifies the molecular mechanism and extends the phenotypic spectrum of the HSAN5 neuropathy. JMed Genet. 2011;48(2):131-135.

57. Larsson E, Kuma R, Norberg A, Minde J, Holmberg M. Nerve growth factor $\mathrm{R} 221 \mathrm{~W}$ responsible for insensitivity to pain is defectively processed and accumulates as proNGF. Neurobiol Dis. 2009;33(2):221-228.

58. Shibuya N, Humphers JM, Fluhman BL, Jupiter DC. Factors associated with nonunion, delayed union, and malunion in foot and ankle surgery in diabetic patients. J Foot Ankle Surg. 2013;52(2):207-211.

59. Hanefeld U, Rees CW, White AJP, Williams DJ. One-pot synthesis of tetrasubstituted pyrazoles-proof of regiochemistry. Journal of the Chemical Society, Perkin Transactions 1. 1996;(13):1545-1552.
60. Martinez MD, Schmid GJ, McKenzie JA, Ornitz DM, Silva MJ. Healing of non-displaced fractures produced by fatigue loading of the mouse ulna. Bone. 2010;46(6):1604-1612.

61. Bouxsein ML, Boyd SK, Christiansen BA, Guldberg RE, Jepsen KJ, Müller R. Guidelines for assessment of bone microstructure in rodents using micro-computed tomography. J Bone Miner Res. 2010;25(7):1468-1486.

62. Webb CM, Cameron EM, Sundberg JP. Fluorescence-labeled reporter gene in transgenic mice provides a useful tool for investigating cutaneous innervation. Vet Pathol. 2012;49(4):727-730. 